\title{
Directed Search for Equilibrium Wage-Tenure Contracts
}

\author{
Shouyong Shi* \\ Department of Economics, Univeristy of Toronto \\ 150 St. George Street, Toronto, Ontario, Canada, M5S 3G7 \\ (email: shouyong@chass.utoronto.ca)
}

This version: October 2007

\begin{abstract}
I analyze the equilibrium in a labor market where firms offer wage-tenure contracts to direct the search of employed and unemployed workers. All workers are identical, every applicant observes all offers, and there is no coordination among individuals. I formulate the equilibrium with directed search and show that it exists. In common with undirected search, wages increase and quit rates decrease with tenure. Moreover, on-the-job search and wage-tenure contracts produce a continuous wage distribution among homogeneous workers. In contrast to undirected search, the applicants choose their targets optimally and separate themselves according to the values of their current contracts. Such endogenous separation generates several novel implications. First, wage mobility is limited in the sense that workers choose to move up on a wage ladder gradually when applying for jobs. Second, the density function of the wage distribution is decreasing at high wages. Third, an increase in the unemployment benefit or the minimum wage has no effect on an employed worker's wage contract and his job-to-job transitions. Finally, equilibrium contracts and employed workers' job-to-job transitions are independent of the distribution of workers. The last feature makes the model tractable for studying business cycles with on-the-job search.
\end{abstract}

JEL classifications: E24, C78, J6.

Keywords: Directed search, On-the-job, Wage-tenure contracts.

* First version: August 2006. I have received useful comments from the participants of the workshops at University of Pennsylvania, Rochester, Yale, UBC, Calgary, the Microfoundations of Markets with Frictions (UQAM, 2007), Research on Money and Markets (Toronto, 2007) and the NBER Summer Institute (2007). Financial support from the Bank of Canada Fellowship and from the Social Sciences and Humanities Research Council of Canada is gratefully acknowledged. The opinion expressed in the paper is my own and it does not reflect the view of the Bank of Canada. All errors are mine. 


\section{Introduction}

Directed search is a matching process in which an individual can use his offer to affect his matching rate. The objective of this paper is to study the equilibrium in a labor market where firms offer wage-tenure contracts to direct workers' search. A wage-tenure contract is a time profile of wages which describes how a worker's wage will evolve with tenure. Employed workers can search on the job. I characterize the equilibrium and establish its existence. The equilibrium yields novel predictions about job-to-job transitions, the wage distribution, and effects of unemployment policy.

To see why directed search is interesting to study, it is useful to contrast it with the large literature on undirected search developed from Diamond (1982), Mortensen (1982), and Pissarides (1990). There are two classes of models in this literature. In one class, as in the three pioneering works, prices (wages) are a result of bargaining after individuals are matched. In the other class, prices are posted but searchers do not know who posted what prices before they are matched (e.g., Burdett and Mortensen, 1998, and Burdett and Coles, 2003). In both classes of models, search is undirected because prices play no role, ex ante, to direct workers to particular matches. Although modelling search as an undirected process is interesting, there are strong reasons for studying directed search. First, some search is directed rather than completely random, particularly for workers who search on the job. For example, applicants often have information about wages from job advertisement, word of mouth, or referrals. Second, undirected search generates an array of market inefficiencies, whose corrective policy depends on the details assumed for the matching and price determination processes (see Hosios, 1990). Directed search can eliminate most of these inefficiencies. Third, wage dispersion in undirected search models is sensitive to the assumption that a worker knows at most one wage before search. For example, the continuous wage distribution in the well-known model of Burdett and Mortensen (1998) would become degenerate if each applicant knew two or more wages. Directed search models do not have this sensitivity because they allow each applicant to observe all offers before the application.

During the last fifteen years or so, a literature has grown to analyze directed search. Peters $(1984,1991)$ and Montgomery (1991) provide two of the earliest formulations. Examples of the further exploration include Moen (1997), Acemoglu and Shimer (1999a,b), Julien, et al. (2000), Burdett, et al. (2001), Shi (2001, 2002), Coles and Eeckhout (2003), Galenianos and Kircher (2005), and Delacroix and Shi (2006). They have shown that an

equilibrium with directed search and its efficiency properties are significantly different from 
those with undirected search.

However, the literature of directed search has its own shortcomings. First, it has ignored wage-tenure contracts and on-the-job search, by assuming that each firm posts a fixed wage for the entire duration of a worker's employment and that a worker must quit his job first before searching for another job. Without wage-tenure contracts, this literature is unable to explain the empirical regularities that wages rise and quit rates fall with tenure (e.g., Farber, 1999). Without on-the-job search, the literature cannot predict job-to-job transitions which constitute a large part of the flow of workers in the data. Second, directed search models generate only a few number (and often a singleton) of equilibrium wages among homogeneous workers, in contrast to the data and to the undirected search model by Burdett and Mortensen (1998). Given the appealing features of directed search discussed above, there is an urgency to advance the theory to bridge these gaps with the data. ${ }^{1}$

To see what is needed to characterize a directed search equilibrium with contracts, let me compare the task with the one in undirected search, which is accomplished by Burdett and Coles (2003, $B C$ henceforth). With undirected search, workers are assumed to send their applications randomly to a pool of recruiting firms. With directed search, however, each worker's application must be optimal in the tradeoff between an offer and the likelihood of obtaining the offer. Similarly, each firm understands that it can raise the offer to entice more workers to apply to it. To describe this tradeoff, an equilibrium must determine two new objects in addition to optimal contracts. One is the employment rate function, which describes how the rate at which an applicant gets an offer varies with the offer. The other is the hiring rate function, which describes how the rate at which a recruiting firm successfully hires a worker varies with the offer. A challenge in characterizing the equilibrium is to show that these functions exist.

I formulate the equilibrium in an environment where all matches have the same productivity, and then establish the existence of the equilibrium. In equilibrium, the hiring rate associated with an offer is indeed an increasing function of the offer, and the employment rate is a decreasing function of the offer. Thus, the tradeoff between an offer and the matching rate is meaningful.

On wage-tenure contracts, the equilibrium with directed search preserves several attractive properties of the undirected search model by BC. First, wages increase and quit rates fall with tenure. Second, there is a baseline contract, where wages start at the lowest equilibrium level and then increase with tenure, and all other equilibrium contracts are

\footnotetext{
${ }^{1}$ Delacroix and Shi (2006) and Julien et al. (2006) are the exceptions that introduce on-the-job search into directed search models, but they do not examine wage-tenure contracts.
} 
sections of this baseline contract with different initial wages. Third, on-the-job search and wage-tenure contracts together generate a continuous wage distribution in the equilibrium, even though all matches have the same productivity. On-the-job search creates jumps in wages when a worker changes the job, while wage-tenure contracts provide smooth increases in wages when a worker stays with a job.

Beyond these similarities, the equilibrium here has little in common with undirected search. A striking difference is that directed search creates the dichotomy that individuals' optimal decisions, equilibrium contracts and the matching rate functions are independent of the distribution of workers. In undirected search models, in contrast, the distribution of workers is critical for determining individuals' optimal decisions. The dichotomy generates novel results of comparative statics. For example, although an increase in the unemployment benefit or a minimum-wage requirement affects the distribution of workers, it has no effect on an employed worker's job-to-job transition rate and his future wage path, given the worker's current wage. Moreover, the dichotomy makes the model tractable for business cycle research with on-the-job search, as I will elaborate in the concluding section.

The second difference is in job-to-job transitions and wage mobility. With directed search, each worker optimally chooses to apply to a unique target which is an increasing function of the worker's state, i.e., the value of the worker's current contract or unemployment benefit. As workers separate themselves this way, wage mobility is endogenously limited by workers' current states despite that there is no difference in productivity across matches. Such limited wage mobility seems realistic (see Buchinsky and Hunt, 1999). In contrast, undirected search models (e.g., BC, 2003, and Burdett and Mortensen, 1998) predict that a worker can receive an offer that lies anywhere in the support of the wage distribution, and that a worker can transit to any wage that lies above his current wage.

The third difference from undirected search is the wage distribution. As said earlier, wage dispersion generated by directed search is robust because all applicants are allowed to observe all offers before they apply. Moreover, the density function of the wage distribution is decreasing at high wages. This feature is an empirical regularity (see Kiefer and Neumann, 1993) which cannot be captured by an undirected search model with homogeneous matches. The equilibrium in the latter model necessarily predicts an increasing density function of equilibrium wages. To modify this prediction, the literature of undirected search has introduced heterogeneity across matches (e.g., van den Berg and Ridder, 1998). It is important to show that directed search can generate a decreasing wage density without such heterogeneity.

Now let me briefly contrast the current paper with Delacroix and Shi (2006), who also 
examine directed search on the job. That paper excludes wage-tenure contracts by assumption. Incorporating wage-tenure contracts allows me not only to explain the patterns of wages and quits over tenure, but also to produce a rich wage distribution. In Delacroix and Shi, the equilibrium wage structure is a wage ladder, the discreteness of which makes the analysis quite messy. Wage-tenure contracts fill in the gap between any two rungs of the ladder, because wages increase with tenure. Moreover, the smoothness enables me to characterize the equilibrium more generally.

To emphasize the differences between directed and undirected search, I maintain four assumptions imposed by BC. First, workers are risk averse; second, the capital market is imperfect so that workers cannot borrow against their future income. These two assumptions are important for generating the wage-tenure relationship, as discussed by BC. Third, a firm does not respond to the employee's outside offers. How reasonable this assumption is varies across different types of markets. ${ }^{2}$ In any case, the assumption is commonly imposed in the literature, and it enables me to compare the results clearly with those in BC. For a model of undirected search without this assumption, see Postel-Vinay and Robin (2002). Finally, all matches have the same productivity and the productivity of a match is public information. For private information or learning about productivity, see Jovanovic (1979), Harris and Holmstrom (1982), and Moscarini (2005). Such productivity differences between matches or over time are clearly important for wage dynamics and turnover, but they are inadequate for explaining the residual wage dispersion in the data. Abstracting from them enables me to have a clear exploration of search frictions.

\section{The Model}

Consider a labor market that lasts forever in continuous time. I normalize the rate of time preference to zero. There is a unit measure of risk averse workers whose utility function is $u(w)$, where $w$ is income. Workers are not able to borrow against their future income, and so the lower bound on wages is 0 . All workers have the same productivity. When employed, each worker produces a flow of output, $y>0$. When unemployed, a worker enjoys a flow of utility, $u(b)$, which is derived from leisure and other benefits in unemployment. However, I will refer to $b$ simply as the unemployment benefit.

\footnotetext{
${ }^{2}$ In many occupations, workers choose to quit for outside offers rather than ask their employers to match the offers. Matching outside offers is more common in other occupations such as economists and professional athletes. However, in these occupations, a main motivation for matching offers might be the competition for workers' ability. Because the current model abstracts from all heterogeneity across matches, the assumption of not responding to outside offers may not be unreasonable.
} 
Assumption 1. The utility function has the following properties: $0<u^{\prime}(w)<\infty$ and $-\infty<u^{\prime \prime}(w)<0$ for all $w \in(0, \infty) ; u^{\prime}(0)=\infty$; and $u(0)=-\infty$.

Risk aversion in the above assumption is not common in the labor search literature, but it is necessary for wage contracts to be non-trivial. The assumption $u(0)=-\infty$ is imposed for the following reason discussed extensively by BC: if $u(0)$ is large, wages may be zero at the beginning of a contract. Although this possibility does not pose serious difficulties to the analysis, it is cumbersome to be included and, hence, excluded by the sufficient condition $u(0)=-\infty$.

All workers face the possibility of death, which arrives at a Poisson rate $\delta \in(0, \infty)$, and a dead worker is replaced with a newborn who enters unemployment. Death is the only exogenous separation, and so employment is permanent until either the worker dies or quits for another job. Burdett and Coles (2003) also model exogenous separation as death, rather than separation into unemployment. The modelling simplifies the analysis by eliminating savings: because an employed worker never returns to unemployment, the worker has no incentive to save provided that wages increase with tenure. ${ }^{3}$

There are a large number of identical and risk-neutral firms that can enter the market. Entry is competitive: every firm can post a vacancy at a flow cost $k>0$. As common in the literature, a firm has a production technology with constant returns to scale and considers different jobs independently. Normalize the production cost to 0 . Recruiting firms announce wage-tenure contracts to compete for workers.

Firms are assumed to commit to the contracts, although a worker can quit his job at any time. This assumption has two requirements. First, as commonly assumed in the search literature, a firm cannot continue to recruit for a filled job in an attempt to replace the current employee or to renegotiate the contract with him. Second, as discussed in the introduction, a firm cannot respond to the employee's outside offers.

A contract specifies a path of wages as a function of the worker's tenure, conditional on the worker's employment in the firm. Because only the lifetime utility generated by a contract matters to a worker, I express a contract alternatively as a path of such utilities, or values. To do so, consider a contract offered at time $s$. Let $V(t, s)$ denote the value of the contract to the worker after a worker has worked under the contract for a length of tenure $t$. This value is the discounted sum of the worker's expected utility generated by the remaining wage path in the contract from time $(s+t)$ onward, conditional on the

\footnotetext{
${ }^{3}$ If there is exogenous separation into unemployment and if workers can save, then only the workers with sufficiently high wages will save against the event of separation when wages increase with tenure. This incentive to save can be eliminated if there are severance pays that increase sufficiently with wages.
} 
worker's optimal quitting strategy in the future. I refer to the value $V(0, s)$ as an offer at time $s$ and the path of values, $\{V(t, s)\}_{t=0}^{\infty}$, as the contract that delivers the offer. Let $w(V(t, s))$ denote the wage level at tenure $t$ according to this contract. I call the function $w(V)$ the wage function.

Throughout this paper, $t$ denotes a worker's tenure rather than the calendar time. To unify the notation, I denote an unemployed worker's "tenure" as $t=\varnothing$. The value for an unemployed worker is denoted $V_{u}=V(\varnothing, s)$ and the unemployment benefit is expressed as $w(V(\varnothing, s))=b$, for all $s$.

All offers are bounded in $[\underline{V}, \bar{V}]$, where

$$
\bar{V}=u(\bar{w}) / \delta, \quad \underline{V}=u(b) / \delta .
$$

$\bar{w}$ is the highest wage which will be determined in Lemma 3.2 and $\bar{V}$ is the lifetime utility of a worker who is employed at the highest wage permanently until death. The lower bound $\underline{V}$ is the lifetime utility of an unemployed worker who is deprived of the opportunity of applying to jobs in the entire lifetime. Because an unemployed worker does have the opportunity to apply for jobs, all equilibrium offers will be strictly higher than $\underline{V}$.

Both unemployed and employed workers can search for jobs. At any instant, a fraction $\lambda_{0}$ of unemployed workers and a fraction $\lambda_{1}$ of employed workers are randomly selected to receive job application opportunities. I allow for the possibility $\lambda_{0}=\lambda_{1}=1$ by letting $\lambda_{0}$, $\lambda_{1} \in(0,1] .{ }^{4}$ A worker who receives the application opportunity observes all firms' offers instantly without any cost and then chooses the offer to apply. As in most search models, a worker can apply to only one offer. ${ }^{5}$

Individuals cannot coordinate their actions. If two or more workers apply to the same offer, the firm randomly selects only one to employ. Thus, the coordination failure generates unemployment. Moreover, if an employed worker gets an offer, the worker must quit his current job before accepting the offer. As discussed in the introduction, the worker's incumbent firm is assumed not to respond to the worker's outside offers. A job is destroyed when the worker either accepts another firm's offer or dies.

\footnotetext{
${ }^{4}$ The $\lambda$ 'es are bounded above by one because they are the fractions of workers who receive job application opportunities at any instant, not the Poisson rates.

${ }^{5}$ Let me clarify two assumptions here. One is that an applicant observes all offers. This assumption is not necessary, because the essential results are the same if each applicant is assumed to observe two offers that are randomly drawn from the offer distribution (see Acemoglu and Shimer, 1999b). The second assumption is that each applicant can apply to only one offer at a time (for multiple applications, see Galenianos and Kircher, 2005). In continuous time, this assumption is not as restrictive as it may sound. Although a worker in reality may be able to send out multiple applications, the probability with which two or more of his applications will be received by different firms at the same instant is negligible.
} 
Because workers observe the offers before they apply, the offers can direct workers' search. Workers and firms both make the tradeoff between an offer and the matching rate at that offer. When making an offer, a firm faces a hiring rate function, $q($.$) . That is,$ a firm knows that it can change the offer to affect its hiring rate directly according to $q($.$) . Similarly, an applicant understands that different offers are associated with different$ employment rates according to an employment rate function, $p($.$) . Because p$ and $q$ are Poisson rates instead of probabilities, they can exceed one.

The functions $q($.$) and p($.$) are equilibrium objects, since they must satisfy two equi-$ librium requirements. First, they must be consistent with aggregation. That is, as firms and workers make their choices under these functions, the resulting matching rates must indeed be given by these functions. Second, the hiring rate function must ensure that the expected profit of recruiting be the same for all equilibrium offers. Delaying the second requirement to section 4 , I specify the first requirement below.

Let me start with a matching function, $\mathcal{M}(\theta, 1)$, which specifies the measure of matches between a measure $\theta$ of workers and a unit measure of firms. Refer to $\theta$ as the tightness. Assume that $\mathcal{M}$ is linearly homogeneous. Given the two functions $p($.$) and q($.$) , individuals'$ decisions induce the tightness, $\theta(V)$, at each value $V$. Aggregate consistency requires that the matching rates satisfy: $q(V)=\mathcal{M}(\theta(V), 1)$ and $p(V)=\mathcal{M}(\theta(V), 1) / \theta(V)$. Using these relationships to eliminate $\theta$, I can express aggregate consistency as $p(V)=M(q(V))$.

The function $M(q)$ embodies all essential properties of the matching function. From now on, I will take $M(q)$ as a primitive of the model and refer to it as the matching function. ${ }^{6}$ To specify the properties of the matching function, let $q(V) \in[\underline{q}, \bar{q}]$ for all $V$, with $0<\underline{q}<\bar{q}$, where $\bar{q}$ is an exogenous upper bound on $q$ given by the matching function and $\underline{q}$ will be restricted by (5.4) later.

Assumption 2. The matching function $M(q)$ satisfies: (i) $M(q)$ is continuous for all $q \in[\underline{q}, \bar{q}]$ and, for all $q$ in the interior of $(\underline{q}, \bar{q})$, the derivatives $M^{\prime}(q)$ and $M^{\prime \prime}(q)$ exist and are finite; (ii) $\bar{q}<\infty$ and $M(\bar{q})=0$; (iii) $M^{\prime}(q)<0$; (iv) $-q M^{\prime \prime}(q) / M^{\prime}(q) \leq 2$.

Part (i) is a regularity condition that is satisfied by many well-known matching functions. Part (ii) is imposed for the convenience of working with bounded functions. Part (iii) is equivalent to $0<\theta \mathcal{M}_{1} / \mathcal{M}<1$, which is satisfied by all matching functions of

\footnotetext{
${ }^{6}$ I follow Moen (1997) and Acemoglu and Shimer (1999a) to take the matching function as given. In contrast, some directed search models have derived the matching function by aggregating agents' strategies, e.g., Peters (1991), Burdett et al. (2001), Julien et al. (2000) and Delacroix and Shi (2006). The main results of the current paper continue to hold when the matching function is endogenized so.
} 
constant returns to scale that are strictly increasing in the arguments. Part (iv) restricts convexity of $M(q)$, which will be useful for ensuring uniqueness of a worker's optimal application decision. ${ }^{7}$ With these assumptions, I will look for an equilibrium with the following properties: $q^{\prime}(V)>0$ and $p^{\prime}(V)<0$.

The following example gives two common matching functions and finds the restrictions for them to satisfy Assumption 2:

Example 2.1. One matching function is the so-called urn-ball matching function. Derived endogenously by Peters (1991) and Burdett et al. (2001), the function has the form: $\mathcal{M}(\theta, 1)=\bar{q}\left(1-e^{-\theta}\right)$ with $\bar{q}<\infty$, which implies:

$$
M(q)=-\frac{q}{\ln (1-q / \bar{q})} .
$$

This function satisfies Assumption 2. Another matching function is the CES function: $\mathcal{M}(\theta, 1)=\left[\alpha \theta^{\rho}+1-\alpha\right]^{1 / \rho}$, where $\alpha \in(0,1)$ and $-\infty<\rho<1$. This function implies:

$$
M(q)=q\left(\frac{q^{\rho}-1}{\alpha}+1\right)^{-1 / \rho}
$$

With this function, parts (i) and (iii) of Assumption 2 are satisfied. Part (ii) is satisfied iff $-\infty<\rho<0$. In this case, $\bar{q}=(1-\alpha)^{1 / \rho}$. Part (iv) is satisfied iff $\alpha \geq 1-(1-\rho) q^{\rho} / 2$. When $\rho \leq-1$, this condition is satisfied for all $\alpha>0$. When $-1<\rho<0$, the condition puts a lower bound on $\alpha$. Note that, for $\rho<0$, the derivative $M^{\prime}(q)$ is unbounded at $q=\bar{q}$.

\section{Workers' and Firms' Optimal Decisions}

In this section, I characterize individuals' optimal decisions and their value functions. Because this paper focuses on stationary equilibria, the time at which a contract is offered does not matter. Thus, unless it is necessary, I suppress the starting time of a contract, $s$, from the notation such as $w(t, s)$ and $V(t, s)$.

\subsection{Optimal Application}

Workers' search is directed by the employment rate function, $p(V)$. This equilibrium object gives the Poisson rate of obtaining an offer $V$ upon application. I will focus on equilibria in which $p($.$) has the properties described in the following claim. The procedure of the$ analysis is to characterize individuals' decisions first by assuming these properties of $p($. and then verify them in the equilibrium (as an implication of Lemma 5.1 later).

\footnotetext{
${ }^{7}$ For a general matching function, part (iv) of the assumption requires $1-\theta \mathcal{M}_{1} / \mathcal{M} \leq$ $\left[-\theta \mathcal{M}_{11} /\left(2 \mathcal{M}_{1}\right)\right]^{1 / 2}$, where the left-hand side is the share of vacancies in the matching function $\mathcal{M}$.
} 
Claim 1. $p(V)$ is bounded, continuous and concave for all $V$. Moreover, $p(V)$ is continuously differentiable and strictly decreasing for all $V<\bar{V}$, with $p(\bar{V})=0$.

Let me analyze the application decision of a worker who has tenure $t$. Refer to the remaining value of the worker's contract, $V(t)$, as the worker's state. (For an unemployed applicant, set $t=\varnothing$ and $V(\varnothing)=V_{u}$.) After receiving a job application opportunity, the expected increase in the value for the worker is:

$$
D(V(t))=\max _{f \in[V(t), \bar{V}]} p(f)[f-V(t)] .
$$

Denote the solution as $f(t)=F(V(t))$. The following lemma describes the main features of the optimal application (see Appendix A for a proof):

Lemma 3.1. $F(\bar{V})=\bar{V}$. For all $V<\bar{V}$, the following results hold: (i) There is a unique and interior solution to (3.1), $f=F(V)$; (ii) $F($.) is continuous and $D(V)$ is differentiable, with $D^{\prime}(V)=-p(F(V))<0$; (iii) $F($.) is strictly increasing; (iv) $F(V)$ obeys:

$$
V=F(V)+\frac{p(F(V))}{p^{\prime}(F(V))} .
$$

Moreover, $\left[F\left(V_{2}\right)-F\left(V_{1}\right)\right] /\left(V_{2}-V_{1}\right) \leq 1 / 2$ for all $V_{2} \neq V_{1}$; (v) If $p($.$) is twice differen-$ tiable, then $F(V)$ is differentiable with $0<F^{\prime}(V) \leq 1 / 2$, and $D(V)$ is twice differentiable.

For a worker at a value $V$, the offer $F(V)$ is the only optimal target of application. Other offers are not optimal for this worker, despite that the worker observes all of them. Offers higher than $F(V)$ have too low employment probabilities to be optimal, while offers lower than $F(V)$ have too low values. Only the offer $F(V)$ provides the optimal tradeoff between the value and the probability of obtaining it.

Not only is a worker's optimal target of application unique, it is also increasing in the current value for the worker. That is, the higher the worker's current value (state), the higher the offer to which the worker will apply. Thus, the applicants choose to separate themselves according to their states. This endogenous separation is optimal because an applicant's payoff function has the single-crossing property with respect to the worker's current value. An applicant's current job is a backup for him when he fails to obtain the applied job. The higher this backup value is, the more the worker can afford to "gamble" on the application and, hence, the higher the offer to which he will apply.

Figure 1 illustrates the single-crossing property in the $(f, p)$-space for two workers, 1 and 2. Worker 1 is at value $V_{1}$ and worker 2 at value $V_{2}$, where $V_{2}>V_{1}$. Worker $i$ 's 
indifference curve can be written as $f=V_{i}+D_{i} / p$, for $i=1,2$. Suppose that the two indifference curves cross each other at a point, $\left(f_{0}, p_{0}\right)$, where $f_{0}>V_{2}$. At this point, the slope of worker $i$ 's indifference curve is $d f / d p<0$, and the absolute value of this slope decreases with $V_{i}$. This implies that the worker with the higher value (worker 2) is more willing to tolerate a low employment probability than does the worker with the low value. Equivalently, to compensate for the same reduction in the probability of getting an offer, worker 2 needs a smaller increase in the offer than worker 1 does.

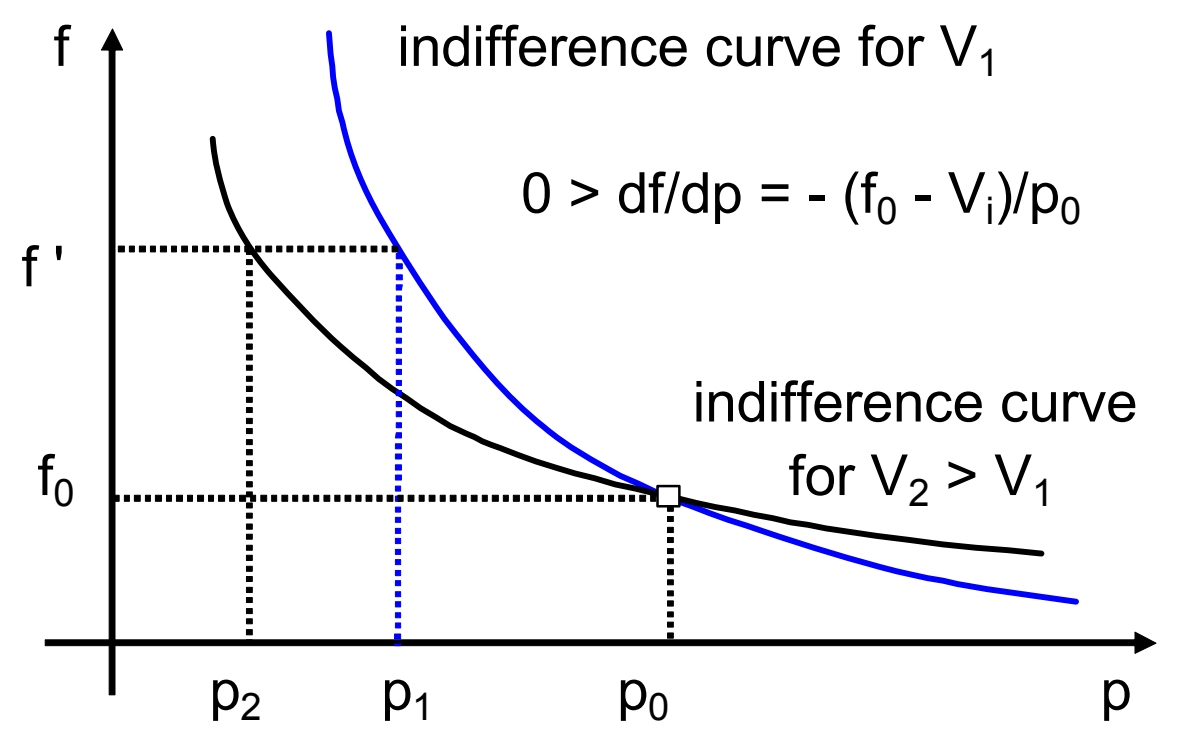

Figure 1. Monotonicity of a worker's optimal application

The optimality of the application decision is one of the key differences between this model and the BC model or, more generally, between directed search and undirected search. By assumption, models with undirected search have no counterpart to the above decision problem of an applicant. This contrast between the two models leads to sharply different predictions on job-to-job transitions and mobility. Directed search predicts a definite pattern of transition and endogenously limited mobility of workers between values or wages. For example, take two workers whose current contracts have remaining values $V_{1}$ and $V_{2}$, respectively, with $V_{1}<V_{2}$. Let $V_{A}=F\left(V_{1}\right)$ and $V_{B}=F\left(V_{2}\right)$. For the two workers, the probability of transiting immediately to a value above $V_{B}$ is zero. Moreover, conditional on that both have transited to new jobs, the likelihood between worker 2's and worker 1's probability of having transited to a value $V^{\prime} \in\left[V_{A}, V_{B}\right)$ is zero. With undirected search, in contrast, the probability of transiting to values above $V_{B}$ is positive for both workers, and the likelihood ratio of transiting to $V^{\prime} \in\left[V_{A}, V_{B}\right)$ is a positive and finite constant.

In addition to limited wage mobility, directed search also yields predictions on the gain to a worker from an application. First, the higher the value of an applicant's current job, 
the less he gains from an application. The expected gain from an application, $D(V)$, and the actual gain in percentage, $(F-V) / V$, both fall as $V$ increases. However, this decreasing gain may not necessarily mean a decreasing gain in wages, because it partly reflects the worker's decreasing marginal utility. Second, $D^{\prime \prime}(V)>0$. That is, the decrease in the expected gain from an application slows down as the worker's current value increases.

\subsection{Value Functions of Workers and Firms}

Throughout this paper, denote $\dot{x}=d x / d t$ for any variable $x$. Recall that $t$ denotes tenure and that an unemployed worker's tenure is denoted as $t=\varnothing$. For an employed worker, the value can change over time for four possible reasons. The first is wage changes with tenure. The second is the event that the worker obtains a better offer and quits the current job. ${ }^{8}$ The third is death. The fourth is the adjustment to the steady state, which is abstracted from by the focus on stationary equilibria. Because the rate of time preference is zero, the value for an employed worker evolves as follows:

$$
\dot{V}(t)=\delta V(t)-u(w(V(t)))-\lambda_{1} D(V(t))
$$

where $D(V)$ is given by (3.1). Since $t$ denotes tenure, $\dot{V}(t)=0$ if wages are constant over tenure. In particular, because the unemployment benefit does not change over time, the value for an unemployed worker (denoted as $V_{u}$ ) obeys:

$$
0=\delta V_{u}-u(b)-\lambda_{0} D\left(V_{u}\right)
$$

Now consider the value function of a firm whose worker has a contract with a remaining value, $V(t)$. Let $J(V(t))$ denote this firm's value. ${ }^{9}$ Because the worker quits at rate $\lambda_{1} p(F(V(t)))$ and dies at rate $\delta$, then

$$
\dot{J}(V(t))=\left[\delta+\lambda_{1} p(F(V(t)))\right] J(V(t))-y+w(V(t))
$$

where $\dot{J}$ denotes the derivative with respect to $t$ rather than $V$. This equation has embodied the aforementioned assumptions that a firm commits to the contract and that it does not respond to the employee's outside offers.

\footnotetext{
${ }^{8}$ The worker can also choose to quit the job to become unemployed if the wage profile is sufficiently decreasing. However, this event will never occur in the equilibrium, because the optimal wage profile has increasing wages with tenure, as shown later.

${ }^{9}$ Strictly speaking, the firm's value at any given tenure $t$ depends on the remaining contract, $\{V(\tau)\}_{\tau \geq t}$, not just on $V(t)$. However, given $V(t)$, the (maximized) value of the firm under an optimal contract that delivers the value $V(t)$ to the worker is a function of $V(t)$ alone. In order to economize on the notation, I treat $J$ as the maximized value of the firm.
} 
For dynamic optimization, let me express $J$ as a discounted sum of profits by integrating (3.5). Since the contracts are expressed in terms of $V$, it is convenient to use $V$ rather than $t$ as the integration variable. To do so, use (3.3) to express:

$$
d t=\frac{d V}{\delta V-u(w(V))-\lambda_{1} D(V)} .
$$

Substituting into (3.5), I obtain a differential equation of $J(V)$. To integrate this equation, let $t_{a}$ be an arbitrary point in $[0, t]$ and let $V_{a}=V\left(t_{a}\right)$. Let $\gamma\left(V(t), V_{a}\right)$ be the probability that a match will survive to tenure $t$ conditional on that it has survived to tenure $t_{a}$. Because the separation rate at any given value $V$ is $\left[\delta+\lambda_{1} p(F(V))\right]$, then

$$
\gamma\left(V, V_{a}\right)=\exp \left[-\int_{V_{a}}^{V} \frac{\delta+\lambda_{1} p(F(m))}{\delta m-u(w(m))-\lambda_{1} D(m)} d m\right],
$$

where I have used (3.6) to substitute $d t$. Equivalently, $\gamma$ is given by the solution to the following differential equation:

$$
\frac{d}{d V} \gamma\left(V, V_{a}\right)=-\frac{\left[\delta+\lambda_{1} p(F(V))\right] \gamma\left(V, V_{a}\right)}{\delta V-u(w(V))-\lambda_{1} D(V)},
$$

where the terminal conditions are $\gamma\left(V_{a}, V_{a}\right)=1$ and $\gamma\left(\bar{V}, V_{a}\right)=0$ for all $V_{a}<\bar{V}$. Because $J$ is bounded, it satisfies the transversality condition: $\lim _{V \rightarrow \bar{V}} J(V) \gamma\left(V, V_{a}\right)=0$ for all $V_{a}<\bar{V}$. Integrating (3.5) with respect to $V$ yields:

$$
J\left(V_{a}\right)=\int_{V_{a}}^{\bar{V}} \frac{[y-w(V)] \gamma\left(V, V_{a}\right)}{\delta V-u(w(V))-\lambda_{1} D(V)} d V .
$$

For any $V_{a}$, this value is determined by the contract remaining at tenure $t_{a}$.

\subsection{Optimal Recruiting Decisions and Contracts}

A firm's recruiting decision contains two parts. The first part is to choose an offer $V(0)$ to maximize the expected value of recruiting, $q(V(0)) J(V(0))$, taking the function $q($.$) as$ given. The second part is to choose a contract $\{w(V)\}_{V=V(0)}^{\bar{V}}$ to deliver the value $V(0)$ and to maximize $J(V(0))$. For the first part, there is a continuum of values that are optimal offers. A high offer attracts more applicants and increases the firm's chance of filling the vacancy, but the ex post profit and value are lower. A low offer yields higher ex post profit and value for the firm, but the chance of filling the vacancy is lower. Different offers in the equilibrium yield the same expected value to a recruiting firm.

For the second part of the decisions, an optimal contract, $\{w(V)\}_{V=V(0)}^{\bar{V}}$, solves:

$(\mathcal{P}) \max J(V(0))$, given $V(0)$. 
Use the notation $V_{0}=V(0)$. Treat $\gamma\left(V, V_{0}\right)$ as an auxiliary state variable and (3.8) as its law of motion. Let $\Lambda$ be the shadow price of $\gamma$. The Hamiltonian of $(\mathcal{P})$ is:

$$
\mathcal{H}\left(V, V_{0}\right)=\frac{y-w(V)-\Lambda\left[\delta+\lambda_{1} p(F(V))\right]}{\delta V-u(w(V))-\lambda_{1} D(V)} \gamma\left(V, V_{0}\right)
$$

Following a similar argument to that in BC, it can be shown that the assumption $u(0)=$ $-\infty$ implies $w(V(t))>0$ for almost all $t$ in all optimal contracts. ${ }^{10}$ In Appendix B, I show that the optimality conditions of the Hamiltonian problem yield: $\Lambda=J$ and

$$
J^{\prime}(V)=-\frac{1}{u^{\prime}(w(V))}<0 .
$$

Optimal contracts have three important properties. First, all optimal contracts provide optimal sharing of the value between a firm and its worker, as described by (3.9). That is, the amount of wage increase that is needed to increase the worker's utility by a marginal unit must be equal to the reduction in the firm's profit. Note that (3.9) can be written equivalently as $-\dot{J}=\dot{V} / u^{\prime}(w)$. For the analysis later, it is useful to substitute $\dot{V}$ from (3.3) and $\dot{J}$ from (3.5) to rewrite this equation as:

$$
u^{\prime}(w)(y-w)+u(w)=u^{\prime}(w)\left[\delta+\lambda_{1} p(F(V))\right] J(V)+\left[\delta V-\lambda_{1} D(V)\right] .
$$

This equation can be directly explained by viewing a match as a joint asset. The left-hand side of the equation measures the flow of "dividends" of the asset, which consists of the firm's profit, evaluated with the worker's marginal utility, and the worker's utility. The right-hand side is the "permanent income" in utils generated by the asset. In particular, the permanent income to the firm is $\left[\delta+\lambda_{1} p(F)\right] J$, which is translated into units of utility with the marginal utility of the worker. The permanent income to the worker is $\left[\delta V-\lambda_{1} D(V)\right]$. The optimal contract requires that the flow of dividends to the joint asset should be equal to the permanent income of the asset.

Second, wages increase with tenure in all optimal contracts. This feature and the bounds on wages are stated as follows (see Appendix B for a proof):

Lemma 3.2. For all $V(t)<\bar{V}$, wages in an optimal contract satisfy:

$$
0<\frac{d w(V(t))}{d t}=\frac{\left[u^{\prime}(w(V))\right]^{2}}{u^{\prime \prime}(w(V))} \lambda_{1} J(V)\left[\frac{d p(F(V))}{d V}\right] .
$$

Moreover, $\bar{w}=y-\delta k / \bar{q}<y, \bar{V}=u(\bar{w}) / \delta, J(\bar{V})=k / \bar{q}>0$, and $q(\bar{V})=\bar{q}(<\infty)$.

\footnotetext{
${ }^{10}$ It can be shown that the program $(\mathcal{P})$ is concave in terms of Gâteaux derivatives in a neighborhood of the optimal contract, and so the optimum is characterized by the optimality conditions. See http://www.chass.utoronto.ca/ shouyong/shi1/dcontractsup.pdf.
} 
There are two forces that make an optimal wage profile increase smoothly with tenure. The first is a firm's incentive to retain a worker by backloading wages, which appears in (3.11) through the feature $d p(F(V)) / d V<0$; the second force is risk aversion, which appears in (3.11) as $u^{\prime \prime}<0$. These forces work as follows. Because a worker cannot commit to the job, the firm can increase the worker's opportunity cost of quitting by backloading wages: as wages rise with tenure, the probability with which the employee can find a better offer elsewhere falls, and so the worker's quit rate falls with tenure. Thus, a rising wage profile is less costly to the firm than a constant wage profile that provides the same expected value to the worker. However, if workers are risk neutral, then the best way for a firm to backload wages is to offer zero wage initially with a promised jump in wages in the future (see Stevens, 2004). Because this jump is not desirable for risk averse workers, the optimal contract has smoothly increasing wages over tenure. To induce a risk-averse worker to accept the increasing wage profile, rather than a flat wage, the firm can give the worker part of the gains from a lower quit rate in the form of a higher offer.

Because wages are increasing with tenure and bounded above, wages in all optimal contracts increase toward the upper bound $\bar{w}$ as $t \rightarrow \infty$. Accordingly, the value for an employed worker converges to $\bar{V}$. This convergence in the value is monotonic, as I will show later in Corollary 5.3. As a result, a firm's value falls with tenure.

The third property is that all optimal contracts are sections of a baseline contract but with different initial values or wages. More precisely, denoting the baseline contract as $\left\{V_{b}(t)\right\}_{t=0}^{\infty}$, then the entire set of optimal contracts is:

$$
\left\{\{V(t)\}_{t=0}^{\infty}: V(t)=V_{b}(t+s) \text { for all } t, \text { where } s \in[0, \infty)\right\} .
$$

That is, relative to the baseline contract, any other optimal contract amounts effectively to crediting the worker with a length of tenure at the start of the contract and, hence, with a higher initial value (or wage). From this initial value, the contract traces out the remaining section of the baseline contract. ${ }^{11}$

The above property follows from the principle of dynamic optimality. To explain why, compare the baseline contract with another contract $c$ given by $\left\{V_{c}(t)\right\}_{t=0}^{\infty}$, with $V_{c}(0)>$ $V_{b}(0)$. Because the values in both contracts increase with tenure toward $\bar{V}$, there exists a time $s$ such that $V_{b}(s)=V_{c}(0)$. If contract $c$ is optimal for delivering the value $V_{c}(0)$ but if it is not the same as the section of the baseline contract from that value onward, the firm

\footnotetext{
${ }^{11}$ It is worth repeating that an offer with a higher value is not necessarily more attractive to an applicant, because the probability of obtaining the offer is lower. A worker's optimal target of application is unique, given the remaining value of his current contract.
} 
that offers the baseline contract can replace the remaining part of the contract from $V_{b}(s)$ onward by contract $c$, with $V_{b}(t)=V_{c}(t-s)$ for all $t \geq s$. This change will increase the firm's expected value, which contradicts the optimality of the baseline contract.

From now on, I focus on the baseline contract and suppress the subscript $b$. Thus, $V(t)$ denotes the remaining value of the baseline contract at tenure $t$, and $w(V)$ denotes the wage level at the point where the remaining value of the baseline contract is $V$.

With the above property, characterizing the set of equilibrium offers is equivalent to tracing out the baseline contract over tenure. Precisely, the set of equilibrium offers, denoted as $\mathcal{V}$, is $\mathcal{V}=\{V(t): t \geq 0\}$. Denote the inverse function of $V(t)$ as $T(V)$; that is,

$$
T(V(t))=t \quad \text { for all } t \geq 0 .
$$

$T(x)$ records the length of tenure required for a worker to increase the value from $V(0)$ to $x$ according to the baseline contract. Clearly, $T^{\prime}(V)=d t / d V$, which is given by (3.6).

\section{Configuration and Definition of the Equilibrium}

To illustrate the configuration of the equilibrium, denote:

$$
v_{0}=V_{u} \text { and } v_{j}=F^{(j)}\left(v_{0}\right), \quad j=1,2, \ldots,
$$

where $F^{(0)}\left(v_{0}\right)=v_{0}$ and $F^{(j)}\left(v_{0}\right)=F\left(F^{(j-1)}\left(v_{0}\right)\right)$. The set of offers in the equilibrium is $\mathcal{V}=[V(0), \bar{V}]$. Note that $V(0)=v_{1}$ and $V(\infty)=\bar{V}$. Because only unemployed applicants apply to $v_{1}$, then $v_{1}=F\left(V_{u}\right)$. Moreover, note that $F\left(V_{u}\right)>V_{u}=\underline{V}+\lambda_{0} D\left(V_{u}\right) / \delta>\underline{V}$. Thus, $v_{1}=F\left(V_{u}\right)>\underline{V}$, which shows that $\mathcal{V}$ is a strict subset of $[\underline{V}, \bar{V}]$.

Figure 2 depicts the career paths of some workers in the equilibrium. There are workers employed at every value in the interval $\left[v_{1}, \bar{V}\right]$. Take a worker who is employed at value $v_{j}$, where $j \geq 1$. The worker applies only to $v_{j+1}$. If he gets the new job, the jump in the value is depicted by the arched arrow from $v_{j}$ to $v_{j+1}$. If the worker does not get the new job, the worker's contract provides smooth increases in the value, which are depicted by the horizontal arrows. After the value has increased, say to $v_{j}+a$, the worker updates the target of the application to $F\left(v_{j}+a\right)$. This process continues until death. Therefore, the value for a worker increases over time as a result of both the jumps created by successful applications and the smooth increases provided by the contracts.

If on-the-job search were prohibited, only one value, $v_{1}$, would be offered in the equilibrium, as in most models of directed search cited in the introduction. If wages were restricted to be constant over tenure, then workers would be employed only in a discrete 
set $\left\{v_{1}, v_{2}, \ldots, \bar{V}\right\}$ because there would be no increase in values to fill the gap between two adjacent levels. Such a model is analyzed by Delacroix and Shi (2006). By filling the gaps, wage-tenure contracts generate an interval of equilibrium offers. Thus, wage-tenure contracts are useful for a directed search model to generate a continuous distribution of wages among homogeneous workers, as well as the wage-tenure relationship.

\section{unemployed employed workers}

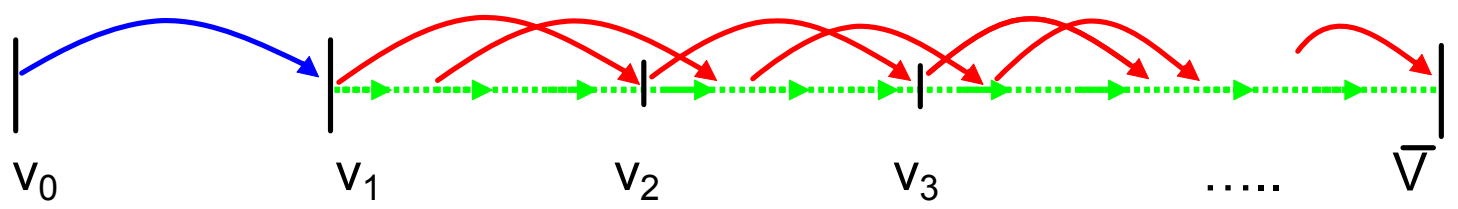

\section{: : unemployed search; : on the job search $\ldots$ : wage increases with tenure according to contracts}

Figure 2. Career paths of some workers

Let $n$ be the fraction of employed workers and $G$ the cumulative distribution function of employed workers over values. As said earlier, wages and values refer to the baseline contract. An equilibrium is a set of offers, $\mathcal{V}=\{V(t): t \geq 0\}$, a Poisson rate of employment, $p($.$) , an application strategy, F($.$) , a value function J($.$) , a wage function w($.$) , and$ the distribution of workers, $(G, n)$, that satisfy the following requirements:

(i) $F(V)$ solves (3.1), given $p($.$) ;$

(ii) Given $F($.$) and p($.$) , each offer V \in \mathcal{V}$ is delivered by a contract that solves

$(\mathcal{P})$ with the starting value $V$, and the resulting value function of the firm is $J(V)$;

(iii) Zero expected profit of recruiting: $q(V) J(V)=k$ for all $V \in[\underline{V}, \bar{V}]$, and $q(V) J(V)<k$ otherwise, where $q(V)=M^{-1}(p(V))$;

(iv) $G$ and $n$ are stationary.

Most elements of this definition are self-explanatory, except (iii). This requirement asks the function $q(V)$ to induce zero expected profit of recruiting for all $V \in[\underline{V}, \bar{V}]$. Because $\mathcal{V}$ is a strict subset of $[\underline{V}, \bar{V}]$, this requirement imposes a restriction on beliefs out of the equilibrium. The reason for imposing this restriction is as follows. For a non-equilibrium value $V \notin \mathcal{V}$, there can be two different reasons why the value is not in the equilibrium 
set. One is the self-fulfilling expectation that no worker will apply to that value: This expectation induces firms not to offer that value, in which case no worker will apply to that value, indeed. The second reason is that, even after firms offer that value, workers still find it optimal not to apply to it. The first reason for a "missing market" may not be robust to trembling that exogenously puts some recruiting firms at the value $V$. Requirement (iii) excludes such non-robust equilibria and, hence, refines the set of equilibria. ${ }^{12}$

Requirement (iii) determines the hiring rate function and, hence, the employment rate function. For given $J($.$) , the requirement yields q(V)=k / J(V)$, and so $p(V)=M(k / J(V))$ for all $V \in[\underline{V}, \bar{V}]$. For all $V>\bar{V}$, (iii) requires that a firm recruiting at $V$ should make an expected loss. This part of the requirement is always satisfied, because Lemma 3.2 implies $q(V) J(V) \leq \bar{q} J(V)<\bar{q} J(\bar{V})=k$.

The above equilibrium definition differs substantially from that in undirected search models, such as Burdett and Mortensen (1998) and BC. As said earlier, a crucial difference is that the application decisions must be optimal in directed search models, while they are random in undirected search models. An important implication of the equilibrium is that the distributions of offers and workers in directed search models play no role in determining individuals' decisions, equilibrium contracts and employed workers' job-to-job transition rates. In particular, parts (i) - (iii) in the above definition, which determine individuals' optimal decisions and equilibrium functions $p($.$) and q($.$) , do not involve the distributions.$

The explanation for why the distributions do not "matter" under directed search is that the workers who apply to any given offer $V$ and the firms that recruit at $V$ form a submarket endogenously. That is, only the workers who are currently at the value $F^{-1}(V)$ will apply to the offer $V$. Different submarkets are connected through the function $p($.$) or$ $q($.$) , which is pinned down by the free-entry condition of firms without any reference to the$ distributions. To illustrate this feature of the equilibrium explicitly, I depict the fixed-point problem of the equilibrium in Figure 3. Given a hiring rate function $q($.$) , consistency with$ the matching function yields the employment rate function $p($.$) . Knowing the function$ $p($.$) is sufficient for the workers to choose the target of optimal application, F($.$) . These$ functions, $p(V)$ and $F(V)$, together determine the quit rate of a worker who is employed at

\footnotetext{
${ }^{12}$ Similar refinements have been used in directed search models, e.g., Acemoglu and Shimer (1999b) and Delacroix and Shi (2006). In Delacroix and Shi, the refinement restricts the applicants', rather than firms', expected payoff off the equilibrium path. It requires a worker's expected surplus from applying to every offer (including a non-equilibrium offer) to be the same. In an environment with homogeneous workers, this alternative restriction achieves the same purpose as requirement (iii) does. However, when the applicants are heterogeneous as in the current model, the alternative restriction is not useful because it is not possible to find one function $p($.$) that induces all applicants to be indifferent between equilibrium$ and non-equilibrium offers.
} 
any given value $V$. For a firm that employs a worker at $V$, the quit rate summarizes all the effects of competition on the firm's expected stream of profit. Thus, given the quit rate, the firm can calculate the expected value under any wage contract and choose the contract optimally. This determines the wage function, $w(V)$, and the firm's value function, $J(V)$. Finally, the free-entry condition ties the loop by determining the hiring rate function $q($. which, in the equilibrium, must be the same as the one the process started with. In this process, the distributions of offers and workers play no role. ${ }^{13}$

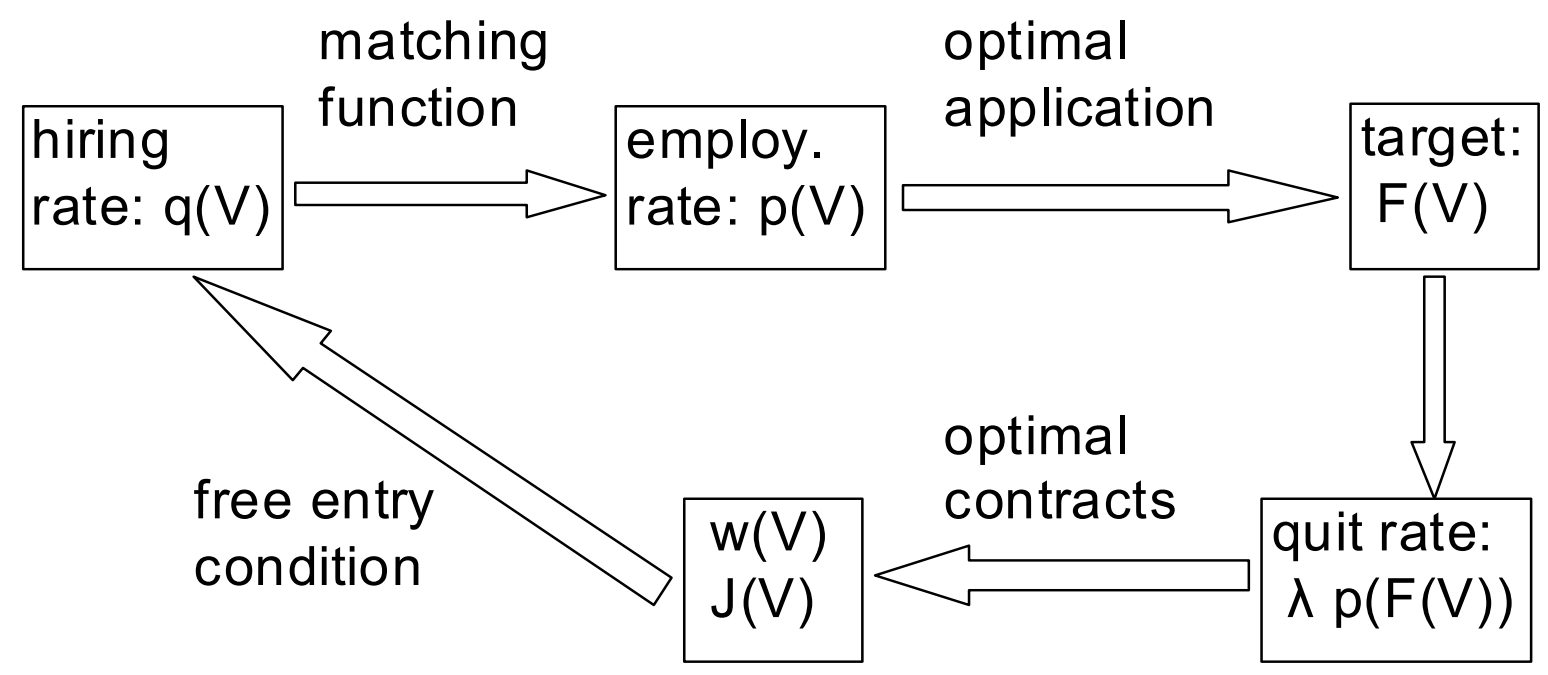

Figure 3. The fixed-point problem

In contrast, models of undirected search do not possess the above independence of contracts and job-to-job transitions on the distributions. First of all, since workers randomly apply to offers in undirected search models, a worker quits his current job whenever he receives a better offer. Hence, the quit rate is a function of the distribution of offers. Second, a firm's hiring rate is a function of the distribution of workers because all the workers whose current values are less than the firm's offer will accept the offer. Thus, the distributions of offers and workers play critical roles in individuals' decisions and equilibrium contracts. In turn, these decisions and contracts affect the flows of workers which determine the distribution of workers through a non-linear partial differential equation. The two-way dependence makes it difficult to characterize an equilibrium in undirected search models, especially when there are shocks to the economy.

Therefore, directed search simplifies the characterization of the equilibrium drastically.

\footnotetext{
${ }^{13}$ If the number of firms is fixed, rather than being determined by free entry, then the expected value of recruiting is endogenous and depends on the distribution of workers. Even in this case, the distribution plays only a limited role because it affects individuals' decisions and the functions $p($.$) and q($.$) entirely$ through the expected value of recruiting.
} 
In the next section, I will determine the equilibrium functions, $p(),. q(),. w($.$) and J($.$) .$ Then, section 6 will solve for the distribution of workers.

\section{Equilibrium Matching Rates and the Wage Function}

To determine the equilibrium functions, $p(),. q(),. w($.$) and J($.$) , I formalize the fixed-point$ problem depicted in Figure 3. Although the process can start anywhere in the loop, it is convenient to organize the analysis around the wage function, $w(V)$. The following procedure develops a mapping on $w$ and obtains $(p, J, F)$.

Start with an arbitrary function $w($.$) and add the subscript w$ to other functions constructed with this given function. First, I integrate (3.9) and use $J(\bar{V})=k / \bar{q}$ to get:

$$
J_{w}(V)=k / \bar{q}+\int_{V}^{\bar{V}} \frac{1}{u^{\prime}(w(z))} d z .
$$

Second, the zero-profit condition for recruiting yields $q_{w}(V)=k / J_{w}(V)$ and, hence,

$$
p_{w}(V)=M\left(\frac{k}{J_{w}(V)}\right) .
$$

Third, using $p_{w}(V)$ as the employment rate, I can express an applicant's optimal decision as $f=F_{w}(V)$ and the expected gain as $D_{w}(V)$.

Fourth, I explore (3.10) to obtain a mapping on $w$. Treat $w$ on the left-hand side of (3.10) as a variable but substitute the given function $w(V)$ for $w$ on the right-hand side. To avoid confusion, use $w_{1}$ instead of $w$ on the left-hand side. Then,

$$
u\left(w_{1}\right)+u^{\prime}\left(w_{1}\right)\left(y-w_{1}\right)=u^{\prime}(w(V))\left[\delta+\lambda_{1} p_{w}\left(F_{w}(V)\right)\right] J_{w}(V)+\delta V-\lambda_{1} D_{w}(V) .
$$

Denote the solution for $w_{1}$ as $w_{1}(V)=(\psi w)(V)$. Equilibrium wage function, $w(V)$, is a fixed point of $\psi$. That is, $w(V)=(\psi w)(V)$ for all $V$. This fixed point is independent of the distribution of workers. So are the functions $p(),. q(),. J($.$) and F($.$) .$

To characterize the fixed point for $w$, let me specify the bounds on various functions. First, using the constant $\bar{w}$ to replace the function $w(V)$ in (5.1) and (5.2), I obtain $J_{\bar{w}}(V)$ and $p_{\bar{w}}(V)$. Because $J_{w}($.$) and p_{w}($.$) are monotone in w$, then $J_{w}(V) \leq J_{\bar{w}}(V)$ and $p_{w}(V) \leq p_{\bar{w}}(V)$ for all $V$. Second, define

$$
\underline{q}=k / J_{\bar{w}}(\underline{V})
$$

Since $J_{\bar{w}}(V)$ is decreasing in $V, \underline{q} \in(0, \bar{q})$ and $q(V) \in[\underline{q}, \bar{q}]$ for all $V$. This lower bound on $q$ is the one used in Assumption 2. Similarly, $p(V)$ is bounded in $[0, M(\underline{q})]$. Third, let $\underline{w}$ be a strictly positive number that is sufficiently close to 0 . 
Assumption 3. Assume that $b, \underline{V}$ and $\underline{w}$ satisfy:

$$
\begin{gathered}
(0<) b<\bar{w}=y-\delta k / \bar{q}, \\
J_{\bar{w}}(\underline{V})\left[\delta+\lambda_{1} p_{\bar{w}}(\underline{V})\right]<y, \\
u(\underline{w})+u^{\prime}(\underline{w})\left[y-\underline{w}-J_{\bar{w}}(\underline{V})\left(\delta+\lambda_{1} p_{\bar{w}}(\underline{V})\right)\right] \geq u(b) .
\end{gathered}
$$

The condition (5.5) is necessary for there to be any worker employed. (5.6) and (5.7) are sufficient conditions to ensure that $\psi w(V) \geq \underline{w}$ for all $V$, given that $w(V) \geq \underline{w}$. The condition (5.6) requires that the permanent income of a job to a firm be less than output even when the firm is providing the lowest value to the worker. Because $J_{\bar{w}}(V)$ and $p_{\bar{w}}(V)$ are decreasing functions and because $\underline{V}$ is an increasing function of $b,(5.6)$ puts a lower bound on $b$. This lower bound is strictly lower than $\bar{w}$ because (5.6) is satisfied when $b=\bar{w}$. (5.7) imposes an upper bound on $\underline{w}$. Because $\underline{w}$ can be chosen to be sufficiently close to 0 and because $b>0$, a sufficient condition for (5.7) is:

$$
\lim _{w \downarrow 0}\left[u(w)+u^{\prime}(w)(a-w)\right]=\infty \text { for all } a>0 .
$$

This sufficient condition is satisfied by the example $u(w)=\left(w^{1-\eta}-1\right) /(1-\eta)$ with $\eta>1$.

Define

$$
\begin{gathered}
\Omega=\{w(V): w(V) \in[\underline{w}, \bar{w}] \text { for all } V ; w(\bar{V})=\bar{w} ; \\
\quad \text { and } w(V) \text { is continuous and (weakly) increasing }\}, \\
\Omega^{\prime}=\{w \in \Omega: w(V) \text { is strictly increasing for all } V<\bar{V}\} .
\end{gathered}
$$

I establish that a fixed point of $\psi$ exists in $\Omega$ and then show that it lies in the subset $\Omega^{\prime}$. First, the following lemma holds (see Appendix B for a proof):

Lemma 5.1. For any $w \in \Omega, J_{w}(V)$ defined by (5.1) is strictly positive, bounded, strictly decreasing and continuously differentiable for all $V$. The function $p_{w}(V)$ defined by (5.2) indeed has all the properties stated in Claim 1.

Because $p_{w}(V)$ has all the properties in Claim 1, parts (i) - (iv) of Lemma 3.1 hold. In particular, there is a unique and interior solution to $(3.1), F_{w}(V)$, which is continuous and strictly increasing for all $V<\bar{V}$. Moreover, $D_{w}^{\prime}(V)=-p_{w}\left(F_{w}(V)\right)<0$.

Theorem 5.2. Maintain Assumptions 1, 2 and 3. Assume that the image of $\psi$ is compact. Then, the mapping $\psi$ has a fixed point $w^{*} \in \Omega^{\prime}$. The implied functions $J_{w^{*}}(V)$ and $p_{w^{*}}(V)$ are strictly concave, in addition to the properties stated in Lemma 5.1. 


\section{Proof. See Appendix C.}

This theorem implies the central properties of wage-tenure and wage-quit relationships. First, wages and values increase with tenure. Second, because $p($.$) is decreasing and F($. is increasing, the quit rate of a worker decreases with tenure and wages. The theorem does not claim that the equilibrium is unique or that the mapping $\psi$ is a contraction. However, in the numerical example in section 7 , the equilibrium is unique.

In the remainder of this paper, I will suppress the asterisk on the fixed point and the subscript $w^{*}$ on the equilibrium functions $J, p, F$ and $D$. Moreover, I will focus on wage profiles that are smooth over tenure. The following corollary describes the additional properties generated by smooth wage profiles (see Appendix D for a proof):

Corollary 5.3. If $w(V(t))$ is a smooth function, i.e., if $|\dot{w}(V(t))|<\infty$ for all $t$, then $w(V)$ is differentiable, with $0<w^{\prime}(V)<\infty$ for all $V$. Moreover, the following results hold for all $V<\bar{V}$ : (i) the derivatives $J^{\prime \prime}(V), p^{\prime \prime}(V)$ and $F^{\prime}(V)$ exist and are finite; (ii) $\dot{V}$ and $\dot{J}$ both exist, with $\dot{V}>0$ and $\dot{J}(V)<0$.

To conclude this section, let me compare wages with unemployment benefits. To do so, let $B(V)$ be the benefit that achieves the value $V$ for an unemployed worker. For any given $V, B(V)$ is the solution for $b$ in (3.4) where $V_{u}$ is replaced with $V$. That is,

$$
B(V)=u^{-1}\left(\delta V-\lambda_{0} D(V)\right) .
$$

Since $D^{\prime}(V)<0$, then $B^{\prime}(V)>0$. Moreover, $B(\bar{V})=\bar{w}$. The following corollary follows from (3.3) and the feature $\dot{V}>0$ for all $V<\bar{V}$ (the proof is omitted):

Corollary 5.4. $w(\bar{V})=B(\bar{V})$. If $\lambda_{0} \leq \lambda_{1}$, then $w(V)<B(V)$ for all $V \in\left[v_{1}, \bar{V}\right)$.

The novel part of this corollary is the case $\lambda_{0}=\lambda_{1}$. In this case, an unemployed worker has the same access to jobs as an employed worker, but the unemployment benefit must be higher than the wage in order for an unemployed worker to achieve the same value $V$ as an employed worker. If the unemployment benefit is the same as (or lower than) an employed worker's wage, the present value for the unemployed worker is lower than that for the employed worker. The reason is that an employed worker enjoys the prospect of rising wages over tenure while an unemployed worker's benefit does not change over time. If an unemployed worker wants to achieve the same value as an employed worker, this 
disadvantage of an unemployed worker must be compensated by a higher unemployment benefit. The result may hold even for some $\lambda_{0}>\lambda_{1} \cdot{ }^{14}$

\section{Equilibrium Distribution of Workers}

Now let me turn to the distribution of workers. Recall that $n$ is the fraction of employed workers and $G$ is the cumulative distribution function of employed workers. Let $g$ be the density function corresponding to $G$. Note that this distribution is over values. Once this distribution is determined, the distribution of employed workers over wages can be deduced from $G_{w}(w(V))=G(V)$, with a density function $g_{w}(w)=g(V) / w^{\prime}(V)$.

To determine the distribution, let me examine the group of workers who are employed at values less than or equal to $V$, where $V \in\left[v_{1}, \bar{V}\right]$. The measure of this group is $n G(V)$. There is only one inflow into this group, which is the flow of workers from unemployment into employment at the value $v_{1}$. In a small interval of time, $d t$, this inflow is $\lambda_{0}(1-n) p\left(v_{1}\right) d t$. There are three flows out of the described group. First, the workers in the group may die, the flow of which is $\delta n G(V) d t$. Second, for the workers whose values lie in $(V-\dot{V} d t, V]$, their contracts increase their values above $V$ after the length of time $(d t)$. The measure of this outflow is $n[G(V)-G(V-\dot{V} d t)]$. Third, some workers in the group quit for offers whose values are higher than $V$. These quitters are currently employed in $\left(F^{-1}(V), V\right]$ if $F^{-1}(V) \geq v_{1}$, and in $\left(v_{1}, V\right]$ if $F^{-1}(V)<v_{1}$. Thus, quitting generates the following measure of outflow from the described group:

$$
(d t) \lambda_{1} n \int_{\max \left\{v_{1}, F^{-1}(V)\right\}}^{V} p(F(z)) d G(z) .
$$

For the distribution of employed workers to be stationary, the flow of workers into the described group must be equal to the sum of the outflows. Imposing this requirement, re-arranging terms and taking the limit $d t \downarrow 0$, I have:

$$
\begin{aligned}
& \lim _{d t \downarrow 0} \frac{G(V)-G(V-\dot{V} d t)}{d t} \\
= & \lambda_{0} \frac{1-n}{n} p\left(v_{1}\right)-\delta G(V)-\lambda_{1} \int_{\max \left\{v_{1}, F^{-1}(V)\right\}}^{V} p(F(z)) d G(z) .
\end{aligned}
$$

To solve for the distribution, partition the support of $G$ into subintervals $\left[v_{j}, v_{j+1}\right)$, where $v_{j}$ is defined by (4.1). Add a subscript $j$ to $g(V)$ and $G(V)$ for $V \in\left[v_{j}, v_{j+1}\right)$. I prove the following theorem in Appendix E:

\footnotetext{
${ }^{14}$ If $\lambda_{0}<\lambda_{1}$, then an unemployed worker has a more difficult access to jobs than an employed worker. This additional reason for $B(V)>w(V)$ is discussed in Burdett and Mortensen (1998).
} 
Theorem 6.1. The fraction of employed workers is:

$$
n=\frac{\lambda_{0} p\left(v_{1}\right)}{\delta+\lambda_{0} p\left(v_{1}\right)}
$$

The distribution of employed workers is continuous for all $V$, with $G\left(v_{1}\right)=0$. The density function is continuous and, for all $V \neq v_{2}, g(V)$ is differentiable. Moreover, $g$ satisfies:

$$
g(V) \dot{V}=\delta[1-G(V)]-\lambda_{1} \int_{\max \left\{v_{1}, F^{-1}(V)\right\}}^{V} p(F(z)) d G(z) .
$$

With $V(0)=v_{1}, T$ in (3.12) and $\gamma$ in (3.7), $g$ can be solved piece-wise as follows:

$$
\begin{gathered}
g_{1}(V) \dot{V}=\delta \gamma\left(V, v_{1}\right) \\
g_{j}(V) \dot{V}-g_{j}\left(v_{j}\right) \dot{v}_{j} \gamma\left(V, v_{j}\right) \\
=\lambda_{1} \int_{v_{j}}^{V} \gamma(V, z) p(z) g_{j-1}\left(F^{-1}(z)\right) d F^{-1}(z)
\end{gathered}
$$

where (6.5) holds for $j \geq 2$. Moreover, $g_{j}\left(v_{j}\right)=\lim _{V \uparrow v_{j}} g_{j-1}(V)$ for all $j$.

The above theorem documents a few features of the equilibrium distribution of employed workers. First, the distribution is non-degenerate and continuous, despite the fact that all workers and all matches are identical. This feature is remarkable because it is not possessed by previous models of directed search. As mentioned in the introduction, previous models of directed search can only produce a small number of equilibrium wages among homogeneous workers, and this number is often one. On the other hand, the model of undirected search by Burdett and Mortensen (1998) can generate a continuous distribution of values or wages, but the distribution becomes degenerate once every worker can observe two or more offers before search. In my model, each searching worker observes all offers before application. Yet, the equilibrium features a continuous distribution of employed values or wages among homogeneous workers.

On-the-job search is the cause of wage dispersion in this model and it exerts two forces on wages. First, by itself, on-the-job search produces the jumps in wages and produces a wage ladder among homogeneous workers. That is, a worker who gets a job earlier will search for a better job than a worker who gets the same job later. Second, workers' search on the job induces firms to provide wage-tenure contracts which create further dispersion among workers' wages. Note that it is wage-tenure contracts that make the wage distribution continuous, because they fill in the gap between two adjacent rungs of the wage ladder created by the direct effect of on-the-job search. 
Second, there is no mass at the lowest value of employment, since $G\left(v_{1}\right)=0$. This feature is remarkable because there is a positive mass of unemployed workers who apply only to $v_{1}$. Despite this concentration of applications, there is no build-up of workers at $v_{1}$. All the workers who are employed at $v_{1}$ will only stay at $v_{1}$ for a very short length of time. Some of them will quit for better jobs or die, while the rest of them will experience wage increases according to the contracts. Thus, the mass of workers at $v_{1}$ is zero in the stationary equilibrium. In fact, there is no mass point anywhere in the support of the distribution. Moreover, the density function is differentiable except at $V=v_{2} \cdot{ }^{15}$

Third, the density function of employed workers can be computed recursively. Starting with $j=1$, one can compute $g_{1}$ from (6.4). Taking the limit $V \uparrow v_{2}$ in the formula yields $g_{2}\left(v_{2}\right)$. Then, setting $j=2$ in (6.5) yields $g_{2}(V)$. Taking the limit $V \uparrow v_{3}$ in the result yields $g_{3}\left(v_{3}\right)$. Continuing this process, one can obtain $g_{j}$ for all $j$.

The following corollary describes the upper tail of the density function (see Appendix E for a proof):

Corollary 6.2. $g(\bar{V})=0$ and $g_{w}(\bar{w})=0$ if and only if $\left|\delta-\lambda_{1} p(\bar{V}) / F^{\prime}(\bar{V})\right| \neq 0$. Under this condition, the density function of employed workers is decreasing at values sufficiently close to $\bar{V}$ or, equivalently, at wages close to $\bar{w}$. Moreover, the CES matching function in Example 2.1 satisfies the condition, $\left|\delta-\lambda_{1} p(\bar{V}) / F^{\prime}(\bar{V})\right| \neq 0$, while the urn-ball matching function satisfies the condition if and only if $\bar{q} \neq \delta / \lambda_{1}$.

The decreasing density function at high wages is a robust feature of the data (see Kiefer and Neumann, 1993). Note that the condition required for $g(\bar{V})=0$ in the corollary is satisfied easily by the two matching functions in Example 2. Also note that undirected search models with homogeneous matches produce an increasing density function (see Burdett and Mortensen, 1998, and BC).

Directed search is able to generating a decreasing density function at high values or wages because workers choose their applications optimally. To see why, consider a worker with a value $V$ with $F(V)<\bar{V}$. This worker observes all offers. For the worker to apply to the target value, $F(V)$, rather than higher offers, higher offers must be sufficiently more difficult to be obtained than the target value. That is, the measure of recruiting firms per applicant must be sufficiently smaller at high values than at the target value. In particular, at values close to the upper bound $\bar{V}$, the measure of recruiting firms per applicant should

\footnotetext{
${ }^{15}$ I thank Guido Menzio for pointing out that the distribution is continuous at $v_{1}$. The density function may fail to be differentiable at $v_{2}$ because offers below $v_{2}$ in the interval $\left(v_{1}, v_{2}\right)$ do not receive applications from employed workers, while offers above $v_{2}$ do.
} 
be close to zero. In turn, as few workers apply to such high values, it is indeed optimal for only few firms to recruit at these values. The measure of workers who succeed in obtaining jobs at values near $\bar{V}$ is close to zero. This feature makes the density function of employed values decreasing near the upper end of the distribution.

In contrast, undirected search models produce an increasing density function. By the assumption of undirected search, an applicant contacts all firms with the same probability. Relative to a low offer, a high offer increases acceptance and retention, without the negative response on the application side that would arise if search were directed. Thus, more firms recruit and more workers are employed at high values than at low values, resulting in an increasing density function. To modify this unrealistic prediction, the literature of undirected search has introduced particular distributions of heterogeneity across matches in workers' or firms' characteristics (e.g., van den Berg and Ridder, 1998).

Let me illustrate more formally why the two models generate different shapes of the upper tail of the density function. With competitive entry, both models require that a firm's hiring rate at any given offer $V$ should satisfy the zero-profit condition: $q(V)=k / J(V)$. Moreover, because the value function of a firm, $J(V)$, is decreasing and concave in both models, $q(V)$ is increasing and convex. However, the link between $q(V)$ and the distribution of employed workers differs between the two models. With undirected search, this link is tight because the acceptance of a firm's offer $V$ can come from any applicant whose current value is below $V$. That is,

$$
q(V)=\lambda_{1} n G(V)+\lambda_{0}(1-n) .
$$

Because $q$ is convex, then $G$ must be convex, which implies that the density function must be increasing. Directed search breaks this link between $q$ and $G$, because the firms offering $V$ only attract the applicants whose current value is $F^{-1}(V)$. In this case, the property of the density function is determined by the applicants' tradeoff between the offer and the employment probability which, as explained above. ${ }^{16}$

One might conjecture that the density function in this model may still be increasing in most parts of the support, despite that it decreases at values close to $\bar{V}$. If this conjecture were true, then the density function in this model would not be different from that in an undirected search model, such as BC, only at such high values. Unfortunately, it is difficult to verify or contradict this conjecture analytically. However, I will provide a numerical example in the next section to illustrate that the conjecture is not supported.

\footnotetext{
${ }^{16}$ In related models of directed search, Delacroix and Shi (2006) and Galenianos and Kircher (2005) also establish the feature that the density function of employed wages is decreasing at high wages. However, they restrict firms' offers to wage levels rather than wage-tenure contracts.
} 


\section{Policy Analysis and a Numerical Example}

In this section, I examine the effects of some labor market policies and then compute an example to illustrate the equilibrium.

Consider separately the following three policies: an increase in the unemployment benefit, $b$, an assistance to unemployed workers that increases their job application opportunity, $\lambda_{0}$, and a minimum-wage requirement, $w \geq w_{\min }$. For the minimum wage to have a nontrivial effect, assume that it is binding in the sense that $w\left(v_{1}\right)<w_{\min }$, where $w\left(v_{1}\right)$ is the starting wage in the baseline contract which delivers the lowest equilibrium value $v_{1}$ in the absence of the minimum wage. The following corollary summarizes the effects of these policies (see Appendix D for a proof):

Corollary 7.1. An increase in $b, \lambda_{0}$ or the minimum wage affects the distribution of workers and increases the lowest offer in the equilibrium, $v_{1}$. Moreover, an increase in $b$ increases the value for unemployed workers, $V_{u}$, and reduces the measure of employed workers, $n$. An increase in $\lambda_{0}$ increases $V_{u}$ and $n$. The minimum wage reduces $n$ and $V_{u}$. However, the three policies do not affect the functions $w(),. F(),. p(),. q(),. J($.$) and, hence,$ they do not affect equilibrium contracts and an employed worker's job-to-job transitions.

Although most of the effects of the three policies on $\left(v_{1}, V_{u}, n\right)$ are intuitive, two effects are worth noting. First, an increase in the unemployment benefit and an assistance to unemployed workers in finding jobs have opposite effects on employment. A higher unemployment benefit reduces employment by making unemployed workers "picky" about offers. An assistance to unemployed workers in finding jobs also makes them "picky", but it increases employment by directly increasing the flow into employment. Second, the minimum wage reduces the value for unemployed workers, despite that it raises the target value to which an unemployed worker applies. The explanation is that such a higher target value is suboptimal for an unemployed worker's application, because it does make the best tradeoff between the offer and the employment probability.

None of the three policies has any effect on employed workers' decisions and their jobto-job transition rates. More precisely, supposing that the policies increase $v_{1}$ to $\hat{v}_{1}$, then the offers in $\left[v_{1}, \hat{v}_{1}\right)$ are no longer equilibrium offers, but the new baseline contract is the section of the original baseline contract that starts at $\hat{v}_{1}$. Thus, conditional on a worker's current value (or wage), the worker's optimal application, the wage-tenure contract and the worker's transition rate to another job are all independent of $b, \lambda_{0}$ and the minimum wage. The reason for this independence is contained in Figure 3. That is, the fixed-point 
problem that determines the functions $q(V), p(V), F(V), J(V)$ and $w(V)$ involves only employed workers and not unemployed workers.

There are two qualifications to this independence result. First, the above policies do affect aggregate flows of workers from one value (or wage) to another, by affecting the distribution of workers. Second, the independence result relies on the assumption that there is no exogenous separation into unemployment. If there were such separation, then the value of unemployment would appear in (3.3) which determines the values for employed workers. Through this appearance the three policies would affect equilibrium contracts and employed workers' job-to-job transition rates.

Despite these qualifications, the independence result in Corollary 7.1 is still interesting. It suggests that, when search is directed, putting resources to the market related to unemployed workers has only limited effects on wage contracts and wage mobility; the more effective way is to directly change the aspects relevant for employed workers such as $\lambda_{1}$. To appreciate this implication, let me contrast it with the prediction of undirected search models, such as BC (2003). Also excluding exogenous separation into unemployment, the $\mathrm{BC}$ model implies that the above policies have direct effects on equilibrium contracts and an employed worker's job-to-job transition. For example, an increase in the unemployment benefit reduces the probability with which a given offer will be accepted by a worker, thereby increasing the equilibrium distribution of offers. As more firms offer high values than before, workers quit more quickly from low-value jobs. In order to mitigate this increase in quits, firms offer contracts in which wages increase more quickly with tenure than before. An increase in $\lambda_{0}$ and the imposition of a minimum-wage requirement have similar effects on equilibrium contracts and job-to-job transition rates.

To conclude the analysis in this paper, let me compute one example. Take the urnball matching function given in Example 2.1 and let the utility function be $u(w)=$ $\left(w^{1-\eta}-1\right) /(1-\eta)$. The parameters are given the following values:

$$
y=10, k=0.5, b=2, \eta=1, \bar{q}=1, \delta=0.1, \lambda_{0}=1=\lambda_{1} .
$$

Since the example is for the purpose of illustration, these parameter values do not have any particular significance. With these parameter values, the highest wage is $\bar{w}=9.95$ and the highest value is $\bar{V} \approx 22.98$. Moreover, $\underline{V}=u(b) / \delta \approx 6$. 93. Set $\underline{w}=1$. I discretize the interval $[\underline{V}, \bar{V}]$ and compute the equilibrium. The equilibrium is unique. ${ }^{17}$ Figures 4 a

\footnotetext{
${ }^{17}$ See http://www.chass.utoronto.ca/ ${ }^{\sim}$ shouyong/shi1/dcontractsup.pdf for the computation procedure. Note that the parameter values do not satisfy (5.6) and (5.7), but the equilibrium still exists.
} 
through $4 \mathrm{c}$ depict the the computed results.

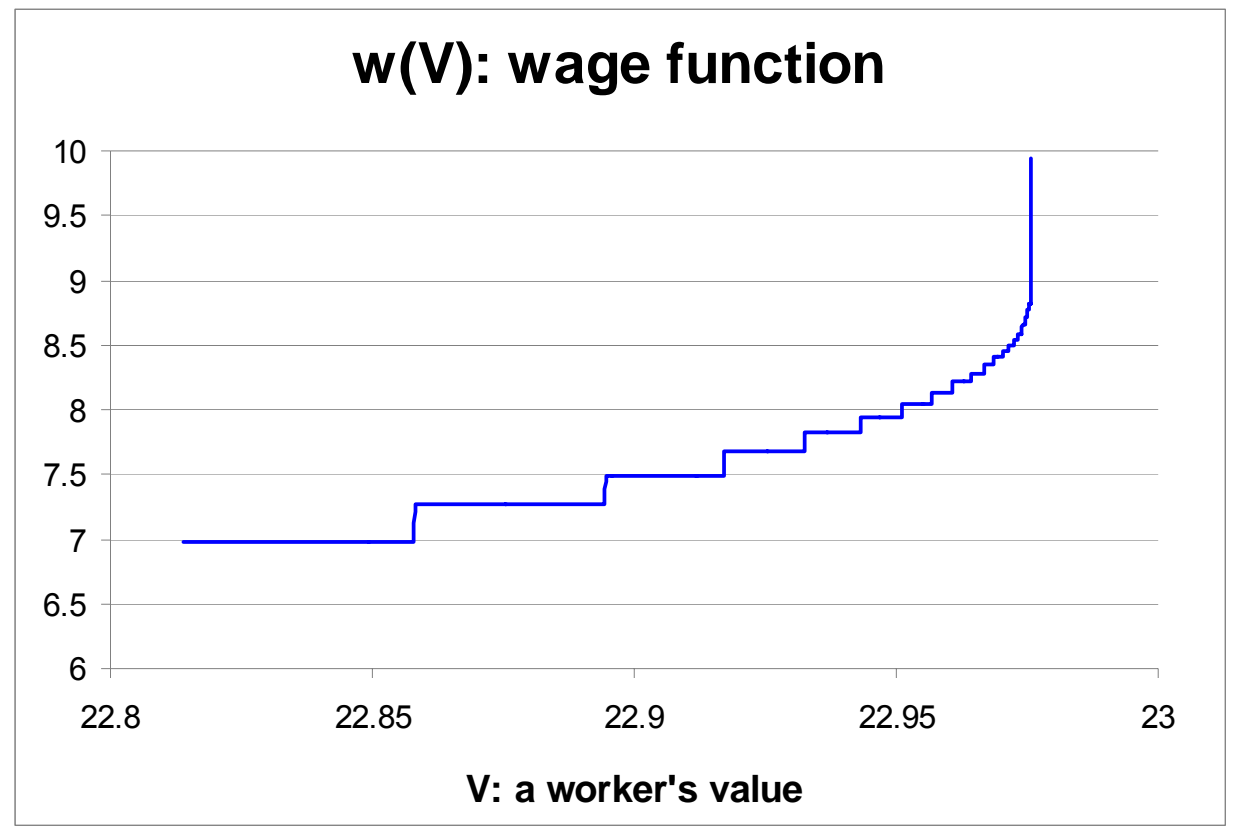

Figure 4a. The wage function in equilibrium contracts

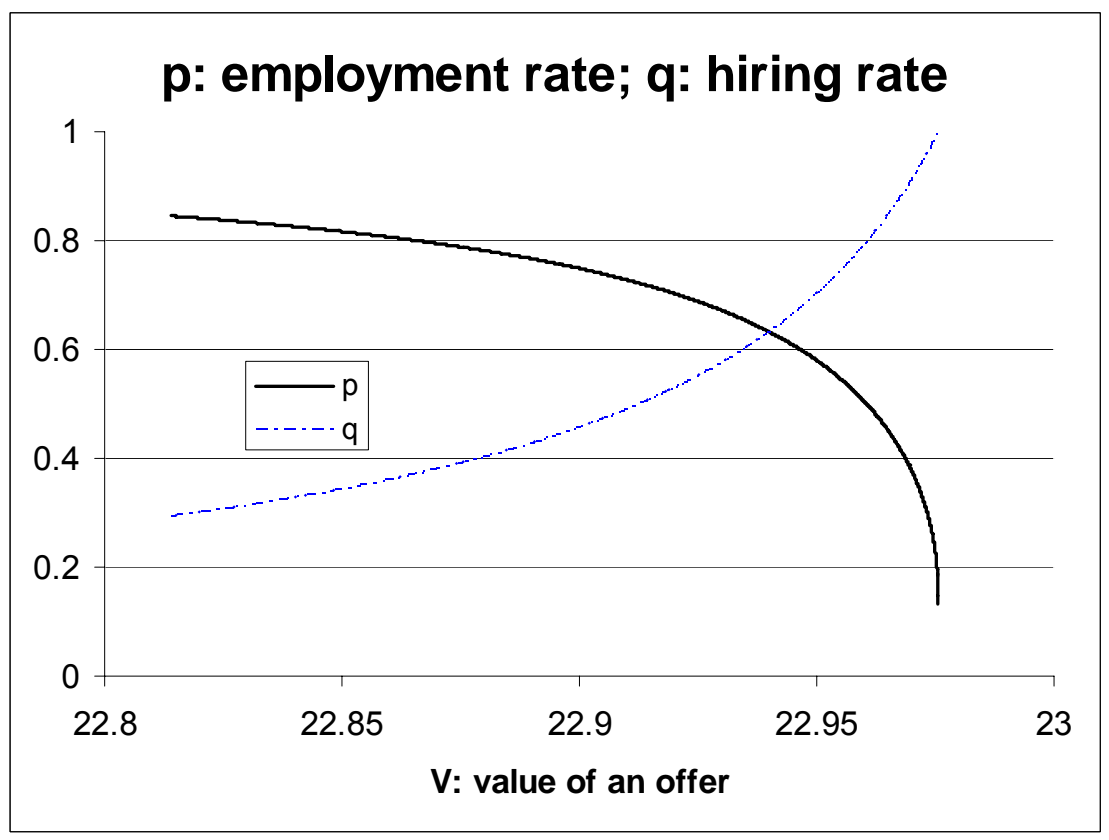

Figure 4b. Employment rates and hiring rates

Figure 4a shows the wage function in the equilibrium. The lowest equilibrium wage is 6.8 , which corresponds to a value $v_{1}=22.79$. The wage function is increasing and convex, implying that wages are increasing with tenure. Figure $4 \mathrm{~b}$ depicts the employment rate 
and the hiring rate, both as functions of the offer value. As proven earlier, the employment rate is a decreasing and concave function, reaching 0 at the highest value $\bar{V}$; the hiring function is an increasing and convex function, reaching $\bar{q}=1$ at the highest value $\bar{V}$.

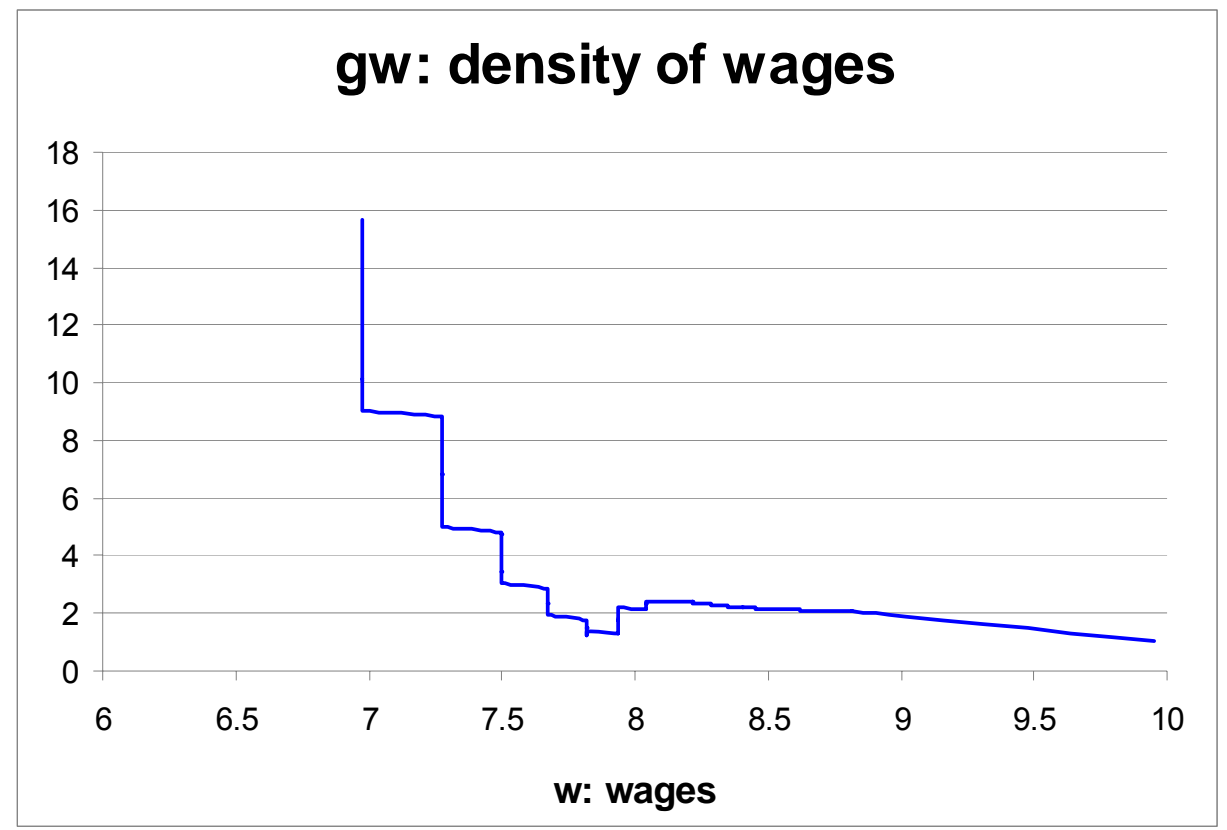

Figure 4c. The density of the distribution of wages

Figure 4c exhibits the density function of the wage distribution. The cumulative function of the wage distribution is a concave, which is not exhibited. The density function is continuous, but discretization in the computation produces the discrete changes in Figure 4c. Note that the density function is decreasing in most parts of the support, in contrast to the increasing function generated by undirected search models. The density function has two sections, divided by the wage level at $w\left(v_{2}\right)=7.822$. At wages below this level, the density function is decreasing. At wages above this level, the density function is first increasing and then decreasing. Thus, the density function is not differentiable at (and only at) the wage level 7.822. As explained before, the non-differentiability is caused by the feature that offers above $v_{2}$ receive the applications of employed workers but offers slightly below $v_{2}$ do not. To eliminate the non-differentiability, one can introduce heterogeneity in the unemployment benefit by assuming that an unemployed worker draws the benefit from a smooth distribution. An earlier version of this paper has introduced such heterogeneity, and it also showed that the density function of the wage distribution can be increasing at low levels of wages. 


\section{Conclusion}

In this paper, I analyze the equilibrium in a labor market where firms offer wage-tenure contracts to direct the search of employed and unemployed workers. All workers are identical, each applicant observes all offers and there is no coordination among individuals. I formulate the equilibrium with directed search on the job and show that it exists. Directed search requires that workers' applications (as well as firms' recruiting decisions) must be optimal, as opposed to random in the literature of undirected search. Because individuals explicitly tradeoff an offer and the matching rate at that offer, each worker chooses a unique offer as the optimal target of the application. Hence, the applicants choose to separate themselves by the values of their current contracts. The applicants for any given offer and the firms that recruit at the offer form a submarket whose only connection to other workers and firms is through an equilibrium function that describes the matching rates associated with different offers.

The endogenous separation of workers and firms generates several implications that are novel in comparison with undirected search. First, wage mobility is limited in the sense that workers choose to move up on a wage ladder gradually when applying for jobs, rather than jumping immediately to very high wages. Second, the density function of the wage distribution is decreasing at high wages, even when all worker-firm pairs are equally productive. Third, an increase in the unemployment benefit or the minimum wage has no effect on an employed worker's wage contract and his job-to-job transition rates. Finally, equilibrium contracts and employed workers' job-to-job transitions are independent of the distributions of offers and workers.

While generating these novel implications, the model preserves several realistic predictions of the undirected search model by BC (2003), without their stringent assumption that each worker can observe at most one offer before applying for a job. First, wages increase with tenure, despite that a worker's productivity is constant over time. Increasing wages with tenure are caused by firms' attempt to backload wages so as to increase retention of the worker in the presence of on-the-job search. Wage increases are smooth because workers are risk averse and cannot borrow against their future income. Second, workers' quit rates fall with tenure, because rising wages with tenure increase the opportunity cost of quitting. Third, there is a continuous wage distribution among homogeneous workers, despite the fact that search is directed.

On-the-job search is the cause of wage dispersion and it works in two ways. First, by itself, on-the-job search produces the jumps in wages and produces a wage ladder among 
homogeneous workers. A worker who gets a job earlier will search for a better job than a worker who gets the same job later. Second, workers' search on the job induces firms to provide wage-tenure contracts which create further wage dispersion. These contracts make the wage distribution continuous, because they fill in the gap between two adjacent rungs of the wage ladder created by the direct effect of on-the-job search.

The most striking feature of the equilibrium is the dichotomy that individuals' decisions and equilibrium contracts are independent of the distribution of workers. This feature makes the model tractable for studying business cycles. It is useful to discuss this tractability in comparison with undirected search models. In the latter models, the distribution is a state variable in every individual's decision problem. Because the distribution evolves endogenously over business cycles, the large dimensionality of the state spaces makes the task of determining the dynamic equilibrium analytically intractable and quantitatively challenging. The dichotomy in my model eliminates this difficulty. Utilizing this feature, Menzio and Shi (2007) examine the implications of on-the-job search on business cycles, by incorporating aggregate and match-specific shocks into the model. 


\section{Appendix}

\section{A. Proof of Lemma 3.1}

The result $F(\bar{V})=\bar{V}$ is evident. Let $V<\bar{V}$ in the following proof. Temporarily denote $K(f, V)=p(f)(f-V)$. Because $p($.$) is continuous and bounded, as stated in Claim$ $1, K(f, V)$ is continuous and bounded. Thus, the maximization problem in (3.1) has a solution. Since all interior values of $f$ yield $K(f, V)>0$, while the choices at the corners yield $K(\bar{V}, V)=0=K(V, V)$ (because $p(\bar{V})=0$ ), then the solution is interior. To show that the solution is unique, I show that $K(f, V)$ is strictly concave in $f$ for all $f \in(V, \bar{V})$. To do so, let $\alpha \in(0,1)$. Let $f_{1}$ and $f_{2}$ be two arbitrary interior values with $f_{2}>f_{1}>V$. Denote $f_{\alpha}=\alpha f_{1}+(1-\alpha) f_{2}$. Then,

$$
\begin{aligned}
K\left(f_{\alpha}, V\right) & =p\left(f_{\alpha}\right)\left[\alpha\left(f_{1}-V\right)+(1-\alpha)\left(f_{2}-V\right)\right] \\
& \geq\left[\alpha p\left(f_{1}\right)+(1-\alpha) p\left(f_{2}\right)\right]\left[\alpha\left(f_{1}-V\right)+(1-\alpha)\left(f_{2}-V\right)\right] \\
& =\alpha K\left(f_{1}, V\right)+(1-\alpha) K\left(f_{2}, V\right)+\alpha(1-\alpha)\left[p\left(f_{1}\right)-p\left(f_{2}\right)\right]\left[f_{2}-f_{1}\right] \\
& >\alpha K\left(f_{1}, V\right)+(1-\alpha) K\left(f_{2}, V\right) .
\end{aligned}
$$

The two equalities come from rewriting, the first inequality from concavity of $p$, and the last inequality from the feature that $p(f)$ is strictly decreasing. Thus, $K(f, V)$ is strictly concave in $f$, which establishes part (i) of the Lemma.

For part (ii), uniqueness of the solution implies that $F($.$) is continuous by the Theorem$ of the Maximum. To show that $D($.$) is differentiable, let V_{1}$ and $V_{2}$ be two arbitrary values with $V_{1}<V_{2}<\bar{V}$. Express $F_{i}=F\left(V_{i}\right)$ for $i=1,2$. Uniqueness of the solution implies $K\left(F_{1}, V_{1}\right)>K\left(F_{2}, V_{1}\right)$ and $K\left(F_{2}, V_{2}\right)>K\left(F_{1}, V_{2}\right)$. Thus,

$$
\begin{aligned}
& D\left(V_{2}\right)-D\left(V_{1}\right)>K\left(F_{1}, V_{2}\right)-K\left(F_{1}, V_{1}\right)=-p\left(F_{1}\right)\left(V_{2}-V_{1}\right) ; \\
& D\left(V_{2}\right)-D\left(V_{1}\right)<K\left(F_{2}, V_{2}\right)-K\left(F_{2}, V_{1}\right)=-p\left(F_{2}\right)\left(V_{2}-V_{1}\right) .
\end{aligned}
$$

Divide the two inequalities by $\left(V_{2}-V_{1}\right)$ and take the limit $V_{2} \rightarrow V_{1}$. Because $F($.$) is$ continuous, the limits show that $D(V)$ is differentiable at $V_{1}$ and that $D^{\prime}\left(V_{1}\right)=-p\left(F_{1}\right)$. Since $V_{1}$ is arbitrary, this argument establishes part (ii).

For part (iii), again take two arbitrary values $V_{1}$ and $V_{2}$, with $V_{1}<V_{2} \leq \bar{V}$. Then, $p\left(F_{j}\right)\left(F_{j}-V_{i}\right)<p\left(F_{i}\right)\left(F_{i}-V_{i}\right)$ for $j \neq i$. I have:

$$
\begin{aligned}
0 & >\left[p\left(F_{2}\right)\left(F_{2}-V_{1}\right)-p\left(F_{1}\right)\left(F_{1}-V_{1}\right)\right]+\left[p\left(F_{1}\right)\left(F_{1}-V_{2}\right)-p\left(F_{2}\right)\left(F_{2}-V_{2}\right)\right] \\
& =p\left(F_{2}\right)\left(V_{2}-V_{1}\right)+p\left(F_{1}\right)\left(V_{1}-V_{2}\right)=\left[p\left(F_{2}\right)-p\left(F_{1}\right)\right]\left(V_{2}-V_{1}\right) .
\end{aligned}
$$

This result implies $p\left(F_{2}\right)<p\left(F_{1}\right)$. Because $p($.$) is strictly decreasing, F\left(V_{2}\right)>F\left(V_{1}\right)$.

For part (iv), note that differentiability of $p$ implies that $F(V)$ is given by the first-order condition, (3.2). Also, because $p$ is concave and decreasing, the following inequalities hold for all $V_{1}$ and $V_{2}$ with $V_{2}>V_{1}$ :

$$
p\left(F_{1}\right) \geq p\left(F_{2}\right)-p^{\prime}\left(F_{1}\right)\left(F_{2}-F_{1}\right),
$$


where $F_{i}=F\left(V_{i}\right), i=1,2$. Substituting this inequality into (3.2) yields:

$$
V_{2}-V_{1} \geq 2\left(F_{2}-F_{1}\right)+p\left(F_{2}\right) \frac{p^{\prime}\left(F_{1}\right)-p^{\prime}\left(F_{2}\right)}{p^{\prime}\left(F_{1}\right) p^{\prime}\left(F_{2}\right)} \geq 2\left(F_{2}-F_{1}\right) \text {. }
$$

This implies $\left(F_{2}-F_{1}\right) /\left(V_{2}-V_{1}\right) \leq 1 / 2$ for all $V_{2} \neq V_{1}$, and so $F$ is Lipschitz.

Finally, for part $(\mathrm{v})$, if $p$ is twice differentiable, then differentiating the first-order condition generates the derivative $F^{\prime}(V)$, and the above Lipschitz property yields $F^{\prime}(V) \leq$ $1 / 2$. In this case, $D^{\prime \prime}(V)=-p^{\prime}(F(V)) F^{\prime}(V)$. QED

\section{B. Proofs of Lemmas 3.2 and 5.1}

I first derive the optimality conditions of the problem $(\mathcal{P})$ and the condition $(3.11)$. From the Hamiltonian of the problem $(\mathcal{P})$, the optimality condition for $\gamma$ is $\mathcal{H} / \gamma=-d \Lambda / d V$ and the optimality condition for $w(V)$ is $\mathcal{H} / \gamma=1 / u^{\prime}(w(V))$. Integrating the condition for $\gamma$ yields $\Lambda=J(V)$, which implies $\mathcal{H} / \gamma=-J^{\prime}(V)$. Substituting into the condition for $w(V)$ yields (3.9). To derive (3.11), use the definition of the Hamiltonian and the optimality condition for $w$ to solve:

$$
\Lambda=\frac{1}{\delta+\lambda_{1} p(F(V))}\left[y-w(V)-\frac{\delta V-u(w(V))-\lambda_{1} D(V)}{u^{\prime}(w(V))}\right] .
$$

Differentiating this equation with respect to $V$ and substituting the result $d \Lambda / d V=-1 / u^{\prime}$, one obtains an expression for $w^{\prime}(V)$. Substituting this expression and (3.3) into $\dot{w}=$ $w^{\prime}(V) \dot{V}$ yields $(3.11)$.

Next, I prove the rest of Lemma 3.2. By Claim 1 and Lemma 3.1, $p^{\prime}(F(V))<0$ and $F^{\prime}(V)>0$ for all $V<\bar{V}$. Because $J(V)>0$ for all $V$, as shown later, then (3.11) implies $\dot{w}(V(t))>0$ for all $V(t)<\bar{V}$. Because $\bar{V}$ is the highest value offered, then $p(F(\bar{V}))=0$ and $\dot{V}=0$ at $V=\bar{V}$. Then $D(\bar{V})=0$, and (3.3) implies $\bar{V}=u(\bar{w}) / \delta$. Similarly, because $\dot{J}(\bar{V})=0,(3.5)$ implies $J(\bar{V})=(y-\bar{w}) / \delta$. Because recruiting at $\bar{w}$ should yield zero net profit, $q(\bar{V}) J(\bar{V})=k$; that is, $\bar{w}=y-\delta k / q(\bar{V})$. If $q(\bar{V})=\bar{q}$, then the stated expressions for $\bar{w}$ and $J(\bar{V})$ follow. Since $\bar{q}<\infty$ by Assumption 2, then $\bar{w}<y$ and $J(\bar{V})>0$.

To show $q(\bar{V})=\bar{q}$, suppose that $q(\bar{V})=\bar{q}-a$ to the contrary, where $a>0$. Because $q(\bar{V}) J(\bar{V})=k>0$ and $J(\bar{V})=(y-\bar{w}) / \delta$, then $\bar{w}=y-\delta k /(\bar{q}-a)$. Consider a firm that deviates from $\bar{w}$ to $\bar{w}+\varepsilon$, where $\varepsilon>0$, which generates a value for a worker as $\hat{V}=u(\bar{w}+\varepsilon) / \delta$. Because the firm is the only one that offers a wage higher than $\bar{w}$, the workers who are employed at $\bar{w}$ will all apply to this firm, which yields $q(\hat{V})=\bar{q}$. The firm's expected value of recruiting is $q(\hat{V}) J(\hat{V})=(y-\bar{w}-\varepsilon) \bar{q} / \delta$, which exceeds $k$ for sufficiently small $\varepsilon>0$. This result contradicts the fact that $\bar{V}$ is an equilibrium value. Thus, $q(\bar{V})=\bar{q}$. This completes the proof of Lemma 3.2.

Now, turn to Lemma 5.1. Let $w(V)$ be an arbitrary function in $\Omega$. It is easy to verify that $J_{w}(V)$ defined by $(5.1)$ is strictly positive, bounded, strictly decreasing and continuously differentiable, with $J^{\prime}(V)=-1 / u^{\prime}(w(V))<0$. Because $w(V)$ is increasing, then $J^{\prime}(V)$ is decreasing and $J(V)$ is (weakly) concave. Moreover, $J_{w}(\bar{V})=k / \bar{q}$. Similarly, 
$p_{w}(V)$ defined by (5.2) is bounded and continuous for all $V$ (including $V=\bar{V}$ ), with $p_{w}(\bar{V})=M(\bar{q})=0$. For all $V<\bar{V}, p_{w}(V)$ is differentiable and strictly decreasing because

$$
p_{w}^{\prime}(V)=\left(M^{\prime} \frac{k}{J_{w}^{2}}\right) \frac{1}{u^{\prime}(w(V))}<0,
$$

where the argument of $M^{\prime}$ is $k / J_{w}(V)$ and where $M^{\prime}<0$ under Assumption 2. Moreover, for any given value $V$,

$$
\frac{d}{d J_{w}}\left(M^{\prime} \frac{k}{J_{w}^{2}}\right)=\frac{k}{J_{w}^{3}}\left(-\frac{k}{J_{w}} M^{\prime \prime}-2 M^{\prime}\right) \geq 0,
$$

where the inequality follows from part (iii) of Assumption 2. Because $J_{w}(V)$ is decreasing and $M^{\prime}<0$, the function $M^{\prime} k / J_{w}(V)$ is decreasing in $V$. Because $1 / u^{\prime}(w(V))$ is increasing in $V$ and $M^{\prime}<0$, then $p_{w}^{\prime}(V)$ is decreasing. That is, $p_{w}(V)$ is (weakly) concave. QED

\section{Proof of Theorem 5.2}

The sets $\Omega$ and $\Omega^{\prime}$ are defined prior to Lemma 5.1 and the mapping $\psi$ is defined by $w_{1}(V)=(\psi w)(V)$, where $w_{1}$ is the solution to (5.3). It can be verified that $\Omega$ is a closed and convex set. Lemmas C.1 and C.2 below state that $\psi: \Omega \rightarrow \Omega^{\prime}$ is a continuous mapping in the supnorm. Under the assumption that the image of $\psi$ is compact, Schauder fixed point theorem implies that $\psi$ has a fixed point in $\Omega$, denoted as $w^{*}$. Because $w^{*}(V)=$ $\left(\psi w^{*}\right)(V) \in \Omega^{\prime}$, then $w^{*}(V)$ is strictly increasing for all $V<\bar{V}$. This implies that $J_{w^{*}}(V)$ and $p_{w^{*}}(V)$ are strictly concave, in addition to the properties stated in Lemma 5.1.

Lemma C.1. $\psi: \Omega \rightarrow \Omega^{\prime} \subset \Omega$.

Proof. Temporarily denote the left-hand side of (5.3) as $L\left(w_{1}\right)$ and the right-hand side as $R(V)$. Recall that $\bar{w}<y$. Because $L(w)$ is continuous and strictly decreasing for all $w<y$, it is invertible for all $w \in[\underline{w}, \bar{w}]$. Then, $w_{1}(V)=L^{-1}(R(V))$. Pick an arbitrary $w \in \Omega$. I show that $w_{1} \in \Omega^{\prime}$. This is done in the following steps.

First, $w_{1}(V)$ is continuous because $J_{w}(),. p_{w}($.$) and F_{w}($.$) are all continuous.$

Second, $w_{1}(V)$ is strictly increasing for all $V<\bar{V}$; i.e., $R(V)$ is strictly decreasing. To establish this result, pick arbitrary values $V_{1}$ and $V_{2}$, with $\underline{V} \leq V_{1}<V_{2}<\bar{V}$, and let $F_{i}=F\left(V_{i}\right)$ with $i=1,2$. I show that the following (stronger) property holds:

$$
0<J_{w}\left(V_{2}\right) S \leq R\left(V_{1}\right)-R\left(V_{2}\right) \leq J_{w}\left(V_{1}\right) S,
$$

where

$$
S \equiv u^{\prime}\left(w\left(V_{1}\right)\right)\left[\delta+\lambda_{1} p_{w}\left(F_{1}\right)\right]-u^{\prime}\left(w\left(V_{2}\right)\right)\left[\delta+\lambda_{1} p_{w}\left(F_{2}\right)\right] .
$$

Note that $S>0$, because $w(V)$ is increasing, $u^{\prime}(w)$ is strictly decreasing, $p_{w}(F)$ is strictly decreasing and $F_{w}(V)$ is strictly increasing. To establish (C.1), note that $J_{w}(V)$ is decreasing and concave with derivative $J_{w}^{\prime}(V)=-1 / u^{\prime}(w(V))<0$. Then,

$$
\frac{V_{2}-V_{1}}{u^{\prime}\left(w\left(V_{1}\right)\right)} \leq J_{w}\left(V_{1}\right)-J_{w}\left(V_{2}\right) \leq \frac{V_{2}-V_{1}}{u^{\prime}\left(w\left(V_{2}\right)\right)} .
$$


Similarly, because the function $\left[\delta V-\lambda_{1} D_{w}(V)\right]$ is increasing and concave with derivative $\left[\delta+\lambda_{1} p_{w}(F)\right]$, I have:

$$
\delta+\lambda_{1} p_{w}\left(F_{1}\right) \geq \frac{\left[\delta V_{2}-\lambda_{1} D_{w}\left(V_{2}\right)\right]-\left[\delta V_{1}-\lambda_{1} D_{w}\left(V_{1}\right)\right]}{V_{2}-V_{1}} \geq \delta+\lambda_{1} p_{w}\left(F_{2}\right) .
$$

Using the first part of the above two results to substitute $J_{w}\left(V_{1}\right)$ and $\left[\delta V_{1}-\lambda_{1} D_{w}\left(V_{1}\right)\right]$ in $R\left(V_{1}\right)$, I get $R\left(V_{1}\right)-R\left(V_{2}\right) \geq J_{w}\left(V_{2}\right) S$. Using the second part of the above two results to substitute $J_{w}\left(V_{2}\right)$ and $\left.\left[\delta V_{2}-\lambda_{1} D_{2}\left(V_{2}\right)\right)\right]$ in $R\left(V_{2}\right)$, I get $R\left(V_{1}\right)-R\left(V_{2}\right) \leq J_{w}\left(V_{1}\right) S$.

Third, $w_{1}(V) \in[\underline{w}, \bar{w}]$ for all $V$, with $w_{1}(\bar{V})=\bar{w}$. Examine $w_{1}(\bar{V})$. Because $w(\bar{V})=\bar{w}$, then (5.3) implies:

$$
L\left(w_{1}(\bar{V})\right)=R(\bar{V})=u^{\prime}(\bar{w})(y-\bar{w})+u(\bar{w})=L(\bar{w}) .
$$

Because $L(w)$ is strictly decreasing, the above equation implies $w_{1}(\bar{V})=\bar{w}$. Since $w_{1}(V)$ is strictly increasing for $V<\bar{V}$, then $w_{1}(V)<\bar{w}$ for all $V<\bar{V}$.

Finally, I show $w_{1}(V) \geq \underline{w}$. Since $L^{\prime}(w)<0, w_{1}(V) \geq \underline{w}$ if and only if $L(\underline{w}) \geq R(V)$. A sufficient condition is $L(\underline{w}) \geq R(\underline{V})$, because $R(V)$ is a decreasing function. Note that the following holds:

$$
\begin{aligned}
R(\underline{V}) & =u^{\prime}(w(\underline{V}))\left[\delta+\lambda_{1} p_{w}\left(F_{w}(\underline{V})\right)\right] J_{w}(\underline{V})+\delta \underline{V}-\lambda_{1} D_{w}(\underline{V}) \\
& <u^{\prime}(\underline{w})\left[\delta+\lambda_{1} p_{w}\left(F_{w}(\underline{V})\right)\right] J_{w}(\underline{V})+u(b) \\
& \leq u^{\prime}(\underline{w})\left[\delta+\lambda_{1} p_{w}(\underline{V})\right] J_{w}(\underline{V})+u(b) \\
& \leq u^{\prime}(\underline{w})\left[\delta+\lambda_{1} p_{\bar{w}}(\underline{V})\right] J_{\bar{w}}(\underline{V})+u(b) .
\end{aligned}
$$

The first inequality follows from the facts that $w(\underline{V}) \geq \underline{w}, \underline{V}=u(b) / \delta$ and $D_{w}(\underline{V})>0$. The second inequality follows from the facts that $F_{w}(\underline{V}) \geq \underline{V}$ and that $p_{w}($.$) is decreasing.$ To obtain the third inequality, note that $J_{w}(V) \leq J_{\bar{w}}(V)$ and $p_{w}(V) \leq p_{\bar{w}}(V)$ for all $V$. Therefore, a sufficient condition for $w_{1}(V) \geq \underline{w}$ is:

$$
L(\underline{w}) \geq u^{\prime}(\underline{w})\left[\delta+\lambda_{1} p_{\bar{w}}(\underline{V})\right] J_{\bar{w}}(\underline{V})+u(b) .
$$

This condition can be re-arranged as (5.7), which is assumed in the Theorem. This completes the proof of Lemma C.1.

Lemma C.2. $\psi$ is continuous in the supnorm.

Proof. To show that the mapping $\psi$ is continuous in the supnorm, I show that the following holds for all $w_{a}, w_{b} \in \Omega$ and all $V$ :

$$
\left|\left(\psi w_{a}\right)(V)-\left(\psi w_{b}\right)(V)\right| \leq A\left\|w_{a}-w_{b}\right\|,
$$

where the norm is the supnorm and $A>0$ is a finite constant. Once this is done, then

$$
\left\|\psi w_{a}-\psi w_{b}\right\|=\sup \left|\left(\psi w_{a}\right)(V)-\left(\psi w_{b}\right)(V)\right| \leq A\left\|w_{a}-w_{b}\right\|,
$$

which implies that $\psi$ is continuous in the supnorm. 
To show (C.2), take arbitrarily $w_{a}, w_{b} \in \Omega$ and $V \in[\underline{V}, \bar{V}]$. Without loss of generality, assume $w_{a}(V) \geq w_{b}(V)$ for the given value $V$. Shorten the subscript $w_{i}$ on $J, p, F$, and $D$ to $i$, where $i=a, b$. Also, denote the right-hand side of $(5.3)$ with $w=w_{i}(V)$ as $R_{i}(V)$. Because $w \geq \underline{w}>0$, Assumption 1 implies that there are positive and finite constants $\omega_{1}$ and $\omega_{2}$ such that $\omega_{1} \leq\left|u^{\prime \prime}(w)\right| \leq \omega_{2}$ for all $w \in[\underline{w}, \bar{w}]$. Then

$$
\left|L^{\prime}(w)\right|=(y-w)\left|u^{\prime \prime}\right| \geq(y-\bar{w}) \omega_{1} \equiv A_{1} .
$$

Note that $A_{1}$ is bounded above 0 . Since $L(w)$ is decreasing, then

$$
\left|R_{a}(V)-R_{b}(V)\right|=\left|L\left(\psi w_{a}(V)\right)-L\left(\psi w_{b}(V)\right)\right| \geq A_{1}\left|\psi w_{a}(V)-\psi w_{b}(V)\right| .
$$

I show that $\left|R_{a}(V)-R_{b}(V)\right| \leq A_{6}\left\|w_{a}-w_{b}\right\|$ for some positive and finite $A_{6}$. Then, (C.2) holds after defining $A=A_{6} / A_{1}$.

To establish the desired inequality for $R$, suppress the given $V$. I have:

$$
\begin{aligned}
& \left|R_{a}-R_{b}\right|=\mid\left\{\left[u^{\prime}\left(w_{a}\right)-u^{\prime}\left(w_{b}\right)\right] J_{a}+u^{\prime}\left(w_{b}\right)\left(J_{a}-J_{b}\right)\right\}\left[\delta+\lambda_{1} p_{a}\left(F_{a}\right)\right] \\
& +\lambda_{1} u^{\prime}\left(w_{b}\right) J_{b}\left[p_{a}\left(F_{a}\right)-p_{b}\left(F_{b}\right)\right]-\lambda_{1}\left[D_{a}-D_{b}\right] \mid \\
& \leq\left[\left|u^{\prime}\left(w_{a}\right)-u^{\prime}\left(w_{b}\right)\right| J_{a}+u^{\prime}\left(w_{b}\right)\left|J_{a}-J_{b}\right|\right]\left[\delta+\lambda_{1} p_{a}\left(F_{a}\right)\right] \\
& +\lambda_{1} u^{\prime}\left(w_{b}\right) J_{b}\left|p_{a}\left(F_{a}\right)-p_{b}\left(F_{b}\right)\right|+\lambda_{1}\left|D_{a}-D_{b}\right| \text {. }
\end{aligned}
$$

I find the bound on each of the absolute values in the above expression.

Because $u^{\prime \prime}<0$, then

$$
\left|u^{\prime}\left(w_{a}\right)-u^{\prime}\left(w_{b}\right)\right| \leq\left|w_{a}-w_{b}\right| \max \left\{\left|u^{\prime \prime}\left(w_{a}\right)\right|,\left|u^{\prime \prime}\left(w_{b}\right)\right|\right\} \leq \omega_{2}\left\|w_{a}-w_{b}\right\| .
$$

By the definition of $J_{w}$,

$$
\begin{aligned}
\left|J_{a}-J_{b}\right| & =\left|\int_{V}^{\bar{V}} \frac{u^{\prime}\left(w_{a}(z)\right)-u^{\prime}\left(w_{b}(z)\right)}{u^{\prime}\left(w_{a}(z)\right) u^{\prime}\left(w_{b}(z)\right)} d z\right| \\
& \leq \frac{1}{\left[u^{\prime}(\bar{w})\right]^{2}} \int_{V}^{\bar{V}}\left|u^{\prime}\left(w_{a}(z)\right)-u^{\prime}\left(w_{b}(z)\right)\right| d z \\
& \leq \frac{\omega_{2}}{\left[u^{\prime}(\bar{w})\right]^{2}} \int_{V}^{\bar{V}}\left|w_{a}(z)-w_{b}(z)\right| d z \leq \frac{\omega_{2}(\bar{V}-\underline{V})}{\left[u^{\prime}(\bar{w})\right]^{2}}\left\|w_{a}-w_{b}\right\| .
\end{aligned}
$$

The coefficient of $\left\|w_{a}-w_{b}\right\|$ is bounded because $u^{\prime}(\bar{w})>0$ and $0<\omega_{2}<\infty$.

To develop bounds on $\left|p_{a}\left(F_{a}\right)-p_{b}\left(F_{b}\right)\right|$ and $\left|D_{a}-D_{b}\right|$, let $\varepsilon=\left\|w_{a}-w_{b}\right\|>0$ with loss of generality. (If $\left\|w_{a}-w_{b}\right\|=0$, then $w_{a}=w_{b}$ for all $V$, in which case $\left|p_{a}\left(F_{a}\right)-p_{b}\left(F_{b}\right)\right|=$ $\left|D_{a}-D_{b}\right|=\left\|w_{a}-w_{b}\right\|$; these provide the required bounds.) I examine two cases separately: the case where $V$ is close to $\bar{V}$ and the case where $V$ is away from $\bar{V}$. The separation is necessary because $M^{\prime}(q)$ and $M^{\prime \prime}(q)$ might be unbounded at $q=\bar{q}$ (i.e., at $V=\bar{V}$ ).

Consider first the case where $V$ is close to $\bar{V}$. In this case, $F_{a}(V)$ and $F_{b}(V)$ are close to $\bar{V}$. Because $p_{w}(V)$ is continuous at $V=\bar{V}$, and because $F(V)$ is continuous, then for given $\varepsilon>0$, there exists $c>0$ such that

$$
\bar{V}-V<c \Longrightarrow\left|p_{i}\left(F_{i}\right)-p_{i}\left(F_{i}(\bar{V})\right)\right|<\varepsilon / 2, \quad \text { for } i \in\{a, b\} .
$$

Because $F_{i}(\bar{V})=\bar{V}$ and $p_{i}(\bar{V})=0$, the following holds for $V>\bar{V}-c$ :

$$
\left|p_{a}\left(F_{a}\right)-p_{b}\left(F_{b}\right)\right| \leq\left|p_{a}\left(F_{a}\right)\right|+\left|p_{b}\left(F_{b}\right)\right|<\varepsilon=\left\|w_{a}-w_{b}\right\|,
$$




$$
\left|D_{a}-D_{b}\right| \leq\left|p_{a}\left(F_{a}\right)\right|\left(F_{a}-V\right)+\left|p_{b}\left(F_{b}\right)\right|\left(F_{b}-V\right)<(\bar{V}-\underline{V})\left\|w_{a}-w_{b}\right\| .
$$

For the last inequality, I used the facts that $\left|p_{i}\left(F_{i}\right)\right|<\varepsilon / 2$ and that $F_{i}-V_{i} \leq \bar{V}-\underline{V}$. (C.5) and (C.6) provide the required bounds when $V>\bar{V}-c$.

Now consider the case where $V \leq \bar{V}-c$, where $c>0$ is constructed above. In this case, $q<\bar{q}$ and, hence, Assumption 2 implies that $\left|M^{\prime}(q)\right|$ and $\left|M^{\prime \prime}(q)\right|$ are bounded for $q \in[\underline{q}, \bar{q})$. Because $p(V)=M\left(\frac{k}{J(V)}\right)$, then

$$
\begin{gathered}
\left|\frac{d M(k / J)}{d J}\right|=\left(-\frac{k}{J^{2}}\right) M^{\prime}\left(\frac{k}{J}\right), \\
\left|\frac{d^{2} M(k / J)}{d J^{2}}\right|=\left(\frac{k}{J_{w}^{3}}\right)\left(-\frac{k}{J_{w}} M^{\prime \prime}-2 M^{\prime}\right) .
\end{gathered}
$$

These absolute values are bounded above in the current case. Let $A_{2}$ and $A_{3}$ be the upper bounds. Define

$$
A_{4}=A_{2} \frac{\omega_{2}(\bar{V}-\underline{V})}{\left[u^{\prime}(\bar{w})\right]^{2}}<\infty .
$$

For any $x \in[\underline{V}, \bar{V}-c]$,

$$
\begin{gathered}
\left|p_{a}(x)-p_{b}(x)\right| \leq A_{2}\left|J_{a}(x)-J_{b}(x)\right| \leq A_{4}\left\|w_{a}-w_{b}\right\|, \\
\left|\frac{d M_{a}}{d J_{a}}-\frac{d M_{b}}{d J_{b}}\right| \leq A_{3}\left|J_{a}-J_{b}\right| .
\end{gathered}
$$

These results lead to the following result:

$$
\begin{aligned}
\left|p_{a}^{\prime}(x)-p_{b}^{\prime}(x)\right| & \leq\left|\frac{d M_{a} / d J_{a}}{u^{\prime}\left(w_{a}\right)}-\frac{d M_{b} / d J_{a}}{u^{\prime}\left(w_{b}\right)}\right| \\
& \leq\left|\frac{d M_{a}}{d J_{a}}\right|\left|\frac{1}{u^{\prime}\left(w_{a}\right)}-\frac{1}{u^{\prime}\left(w_{b}\right)}\right|+\frac{1}{u^{\prime}\left(w_{b}\right)}\left|\frac{d M_{a}}{d J_{a}}-\frac{d M_{b}}{d J_{b}}\right| \\
& \leq \frac{A_{2}}{\left[u^{\prime}(\bar{w})\right]^{2}}\left|u^{\prime}\left(w_{a}\right)-u^{\prime}\left(w_{b}\right)\right|+\frac{A_{3}}{u^{\prime}(\bar{w})}\left|J_{a}-J_{b}\right| \\
& \leq \frac{A_{4}}{V-\underline{V}}|| w_{a}-w_{b}\left\|+\frac{A_{4} A_{3} / A_{2}}{u^{\prime}(\bar{w})}\right\| w_{a}-w_{b} \| .
\end{aligned}
$$

Suppose first that $F_{a} \geq F_{b}$. If $p_{a}\left(F_{a}\right) \geq p_{b}\left(F_{b}\right)$, then

$$
0 \leq p_{a}\left(F_{a}\right)-p_{b}\left(F_{b}\right) \leq p_{a}\left(F_{a}\right)-p_{b}\left(F_{a}\right) \leq A_{4}\left\|w_{a}-w_{b}\right\| .
$$

The second inequality comes from the fact that $p$ is decreasing and the last inequality from the bound on $\left|p_{a}-p_{b}\right|$ just derived. If $p_{a}\left(F_{a}\right)<p_{b}\left(F_{b}\right)$, then

$$
\begin{aligned}
0 & <p_{b}\left(F_{b}\right)-p_{a}\left(F_{a}\right)=-p_{b}^{\prime}\left(F_{b}\right)\left(F_{b}-V\right)+p_{a}^{\prime}\left(F_{a}\right)\left(F_{a}-V\right) \\
& \leq\left(F_{a}-V\right)\left[p_{a}^{\prime}\left(F_{a}\right)-p_{b}^{\prime}\left(F_{b}\right)\right] \leq(\bar{V}-\underline{V})\left[p_{a}^{\prime}\left(F_{b}\right)-p_{b}^{\prime}\left(F_{b}\right)\right] \\
& \leq\left[1+\frac{A_{3}(\bar{V}-V)}{A_{2} u^{\prime}(\bar{w})}\right] A_{4}\left\|w_{a}-w_{b}\right\| .
\end{aligned}
$$

The equality follows from the first-order condition for $F$, the second inequality from the supposition $F_{a} \geq F_{b}$, the third inequality from the facts that $p^{\prime}$ is a decreasing function 
and that $F_{a}-V \leq \bar{V}-\underline{V}$, and the last inequality from the bound on $\left|p_{a}^{\prime}-p_{b}^{\prime}\right|$. Thus, if $F_{a} \geq F_{b}$, then

$$
\left|p_{a}\left(F_{a}\right)-p_{b}\left(F_{b}\right)\right| \leq\left[1+\frac{A_{3}(\bar{V}-\underline{V})}{A_{2} u^{\prime}(\bar{w})}\right] A_{4}\left\|w_{a}-w_{b}\right\| .
$$

Suppose now that $F_{a}<F_{b}$. By switching the roles of $F_{a}$ and $F_{b}$, it can be shown that (C.7) continues to hold. Thus, (C.7) holds for arbitrary $F_{a}(V)$ and $F_{b}(V)$ with $V \leq \bar{V}-c$.

Now let us examine $\left|D_{a}-D_{b}\right|$ for the case $V \leq \bar{V}-c$. If $D_{a} \geq D_{b}$, then

$$
\begin{aligned}
0 & \leq D_{a}-D_{b}=p_{a}\left(F_{a}\right)\left(F_{a}-V\right)-p_{b}\left(F_{b}\right)\left(F_{b}-V\right) \\
& \leq p_{a}\left(F_{a}\right)\left(F_{a}-V\right)-p_{b}\left(F_{a}\right)\left(F_{a}-V\right) \\
& =\left(F_{a}-V\right)\left[p_{a}\left(F_{a}\right)-p_{b}\left(F_{a}\right)\right] \leq(\bar{V}-\underline{V}) A_{4}\left\|w_{a}-w_{b}\right\|
\end{aligned}
$$

The first equality comes from the definition of $D(V)$, the second inequality from the fact that $p_{b}(f)(f-V)$ is maximized at $f=F_{b}$, the last inequality from the bound on $\left|p_{a}-p_{b}\right|$ derived above and the fact $F_{a}-V \leq \bar{V}-\underline{V}$. The same result holds if $D_{a}<D_{b}$. Thus,

$$
\left|D_{a}-D_{b}\right| \leq(\bar{V}-\underline{V}) A_{4}\left\|w_{a}-w_{b}\right\|
$$

Defining $A_{5}=\max \left\{A_{4}, 1\right\}$ and replace $A_{4}$ in (C.7) and (C.8) with $A_{5}$. The resulting bounds on $\left|p_{a}-p_{b}\right|$ and $\left|D_{a}-D_{b}\right|$ apply for both $V>\bar{V}-c$ and $V \leq \bar{V}-c$. Substituting these bounds, (C.3) and (C.4), I have:

$$
\begin{aligned}
\left|R_{a}-R_{b}\right| \leq & \left\{\left[\omega_{2} J_{a}+u^{\prime}\left(w_{b}\right) \frac{A_{4}}{A_{2}}\right]\left[\delta+\lambda_{1} p_{a}\left(F_{a}\right)\right]\right. \\
& \left.+\lambda_{1} A_{5}\left[u^{\prime}\left(w_{b}\right)\left(1+\frac{A_{3}(\bar{V}-\underline{V})}{A_{2} u^{\prime}(\bar{w})}\right) J_{b}+\lambda_{1}(\bar{V}-\underline{V})\right]\right\}\left\|w_{a}-w_{b}\right\| .
\end{aligned}
$$

Let $A_{6}$ be the maximum value of the coefficient of $\left\|w_{a}-w_{b}\right\|$ in the above expression, taken over $V \in[\underline{V}, \bar{V}]$. Then, $A_{6}$ is bounded above. Setting $A=A_{6} / A_{1}$ establishes the inequality (C.2), which shows that $\psi$ is continuous in the supnorm. This completes the proof of Lemma C.2 and, hence, of Theorem 5.2. QED

\section{Proofs of Corollary 5.3 and Corollary 7.1}

To prove Corollary 5.3, suppose that $|\dot{w}(V(t))|<\infty$ for all $t$. If $\dot{V} \neq 0$, then $w^{\prime}(V)=\dot{w} / \dot{V}$ exists and is finite. If $\dot{V}=0$ at some value $V_{c}$, such as $\bar{V}$, then $\delta V_{c}-u\left(w\left(V_{c}\right)\right)-\lambda_{1} D\left(V_{c}\right)=0$. Differentiating this equation with respect to $V_{c}$ yields:

$$
w^{\prime}\left(V_{c}\right)=\frac{\delta+\lambda_{1} p\left(F\left(V_{c}\right)\right)}{u^{\prime}\left(w\left(V_{c}\right)\right)} \in(0, \infty) .
$$

That is, $w(V)$ is differentiable at $V_{c}$. Thus, $w^{\prime}(V)$ exists and is finite for all $V$. From (5.1), (5.2) and Lemma 3.1, one can then verify that $J^{\prime \prime}(V), p^{\prime \prime}(V)$ and $F^{\prime}(V)$ all exist and are finite for all $V<\bar{V}$.

I still need to show that $w^{\prime}(V)>0, \dot{V}>0$ and $\dot{J}(V)<0$ in the case $V<\bar{V}$. In this case, $F(V)<\bar{V}$. Lemma 3.1 implies $d p(F(V)) / d V<0$. The right-hand side of (3.11) 
is positive and finite, which implies $\dot{w}(V)>0$. Thus, $w^{\prime}(V) \dot{V} \in(0, \infty)$ for all $V<\bar{V}$. Because $w(V)$ is strictly increasing for all $V<\bar{V}$ and $\dot{V}$ is bounded (see (3.3)), then $w^{\prime}(V) \in(0, \infty)$ and $\dot{V} \in(0, \infty)$ for all $V<\bar{V}$. Finally, $\dot{J}(V)=J^{\prime}(V) \dot{V} \in(0, \infty)$ for all $V<\bar{V}$. This completes the proof of Corollary 5.3.

To prove Corollary 7.1, note that the analysis in section 5 is independent of $b, \lambda_{0}$ and $G$. Thus, the functions, $w(),. p(),. q(),. J(),. F($.$) , and D($.$) do not change with b$ or $\lambda_{0}$. Similarly, the minimum wage requirement does not affect these functions. However, all three policies affect the distribution of workers and, in particular, affects $n$. The increase in $b$ or $\lambda_{0}$ increases $V_{u}$ (see (3.4)). Since $v_{1}=F\left(V_{u}\right)$, then $v_{1}$ increases with $b$ and $\lambda_{0}$. Clearly, $d\left[\lambda_{0} p\left(v_{1}\right)\right] / d b<0$, and so (6.2) implies that $n$ decreases with $b$. Calculating $d V_{u} / d \lambda_{0}$ from (3.4), calculating $p^{\prime}\left(v_{1}\right)$ from (3.2) and using the fact that $0<F^{\prime}\left(V_{u}\right)<1 / 2$ in Lemma 3.1, it can be shown that $d\left[\lambda_{0} p\left(v_{1}\right)\right] / d \lambda_{0}>0$. Thus, (6.2) implies that $n$ increases with $\lambda_{0}$.

Similarly, the (binding) minimum-wage requirement increases $v_{1}$ and reduces $n$. To show that the requirement reduces $V_{u}$, let $\hat{V}_{u}$ be the new level of $V_{u}$ after the imposition of the requirement and $\hat{v}_{1}$ be the new level of $v_{1}$, where $\hat{v}_{1}>v_{1}$ and $w\left(\hat{v}_{1}\right)=w_{\min }$. Suppose $\hat{V}_{u} \geq V_{u}$, to the contrary. Then,

$$
u(b)=\delta \hat{V}_{u}-\lambda_{0} p\left(\hat{v}_{1}\right)\left[\hat{v}_{1}-\hat{V}_{u}\right] \geq \delta V_{u}-\lambda_{0} p\left(\hat{v}_{1}\right)\left[\hat{v}_{1}-V_{u}\right]>\delta V_{u}-\lambda_{0} D\left(V_{u}\right) .
$$

The equation comes from adapting (3.4) for $\hat{V}_{u}$, the first inequality from the supposition that $\hat{V}_{u} \geq V_{u}$, and the second inequality from the fact that the function $p(v)\left(v-V_{u}\right)$ is decreasing in $v$ for $v>v_{1}$. The above result contradicts (3.4), and so $\hat{V}_{u}<V_{u}$. QED

\section{E. Proofs of Theorem 6.1 and Corollary 6.2}

First, I derive (6.2). Set $V=\bar{V}$ in (6.1). Because $\dot{V}=0$ at $V=\bar{V}$, the left-hand side of (6.1) is equal to 0 at $V=\bar{V}$. Moreover, the integral in (6.1) is equal to zero, because $F^{-1}(\bar{V})=\bar{V}$. Thus, at $V=\bar{V},(6.1)$ yields $(6.2)$.

Second, I show that $G$ is continuous; i.e., $G$ does not have a mass point. Suppose, to the contrary, that $G$ has a mass $m>0$ at some value $V \in\left[v_{1}, \bar{V}\right]$. Then, $G(V)-G(V-\dot{V} d t) \geq$ $m$ for all $d t>0$, and so the left-hand side of (6.1) is equal to $\infty$. This is a contradiction, because the right-hand side of (6.1) is bounded.

Third, the density function, $g$, is continuous for all $V$ and obeys (6.3). To establish continuity of $g$, denote the derivative of $G$ from the left-hand side of $V$ as $g\left(V_{-}\right)$. The left-hand side of $(6.1)$ is equal to $g\left(V_{-}\right) \dot{V}$. Because $G, F, F^{-1}$ and $p($.$) are continuous, the$ right-hand side of (6.1) is continuous in $V$. Thus, $g\left(V_{-}\right) \dot{V}$ must be continuous. Because $\dot{V}$ is continuous, $g$ must be continuous. Then, I can express the left-hand side of (6.1) as $g(V) \dot{V}$. After substituting $p\left(v_{1}\right)$ from (6.2), (6.1) becomes (6.3).

Fourth, $g$ is continuously differentiable for all $V \neq v_{2}$. To see this, note that $F, F^{-1}$ and $p($.$) are continuously differentiable. Since g$ is continuous, $G$ is continuously differentiable, and so the right-hand side of (6.3) is continuously differentiable for all $V \neq v_{2}$. Thus, the left-hand side of the equation, $g(V) \dot{V}$, must be continuously differentiable for all $V \neq v_{2}$. Because $\dot{V}$ is continuously differentiable, $g(V)$ is continuously differentiable for all $V \neq v$. 
Fifth, I derive (6.4). For $V \in\left(v_{1}, v_{2}\right), F^{-1}(V)<v_{1}$, and so (6.3) becomes:

$$
g_{1}(V) \dot{V}=\delta[1-G(V)]-\lambda_{1} \int_{v_{1}}^{V} p(F(z)) g_{1}(z) d z
$$

Because $G\left(v_{1}\right)=0$ by continuity of $G$, taking the limit $V \downarrow v_{1}$ in (E.1) leads to $g_{1}\left(v_{1}\right) \dot{v}_{1}=\delta$. Differentiating (E.1) with respect to $V$ and using (3.8), I get:

$$
\frac{d}{d V}\left[\frac{\dot{V} g_{1}(V)}{\gamma\left(V, v_{1}\right)}\right]=0
$$

Note that $g_{1}\left(v_{1}\right) \dot{v}_{1}=\delta$ and $\gamma\left(V, v_{1}\right) / \gamma\left(z, v_{1}\right)=\gamma(V, z)$. Integrating (E.2) from $v_{1}$ to $V$ yields (6.4). Since $g$ is continuous, taking the limit $V \uparrow v_{2}$ in (6.4) gives $g\left(v_{2}\right)$.

Finally, I derive (6.5) by examining the case $V \in\left[v_{j}, v_{j+1}\right)$, where $j \geq 2$. In this case, $F^{-1}(V) \geq v_{1}$, and so $(6.3)$ becomes

$$
g_{j}(V) \dot{V}=\delta[1-G(V)]-\lambda_{1} \int_{F^{-1}(V)}^{v_{j}} p(F(z)) g_{j-1}(z) d z-\lambda_{1} \int_{v_{j}}^{V} p(F(z)) g_{j}(z) d z .
$$

On the right-hand side of the equation, I have separated the two groups of applicants who successfully obtained jobs with values above $V$ : one coming from $\left(F^{-1}(V), v_{j}\right]$ and the other from $\left[v_{j}, V\right]$. Differentiating (6.3) with respect to $V$ and rewriting the result, I obtain the following equation similar to (E.2):

$$
\frac{d}{d V}\left[\frac{\dot{V} g_{j}(V)}{\gamma\left(V, v_{1}\right)}\right]=\frac{\lambda_{1} p(V)}{\gamma\left(V, v_{1}\right)} g_{j-1}\left(F^{-1}(V)\right) \frac{d F^{-1}(V)}{d V}
$$

Integrating this equation from $v_{j}$ to $V$ yields (6.5). Because $g$ is continuous, then $g_{j}\left(v_{j}\right)=$ $\lim _{V \uparrow v_{j}} g_{j-1}(V)$, all $j$. This completes the proof of Theorem 6.1.

Now, turn to Corollary 6.2. Because $g_{w}(w)=g(V) / w^{\prime}(V)$ and $w^{\prime}(V)>0$ (see Lemma $5.3)$, the property of $g(\bar{V})$ stated in the corollary implies the property of $g_{w}(\bar{w})$. Thus, it suffices to establish the property of $g(\bar{V})$. For this purpose, examine $(6.3)$ at $\hat{V}=\bar{V}-\varepsilon$, where $\varepsilon>0$ is a sufficiently small number. Setting $V=\hat{V}$ in (6.3), dividing the equation by $\varepsilon$ and taking the limit $\varepsilon \rightarrow 0$, I get:

$$
g(\bar{V}) \lim _{\varepsilon \rightarrow 0}\left(\left.\frac{1}{\varepsilon} \dot{V}\right|_{V=\hat{V}}\right)=\delta g(\bar{V})-\lambda_{1} p(\bar{V}) g(\bar{V}) \lim _{\varepsilon \rightarrow 0}\left\{\frac{1}{\varepsilon}\left[\hat{V}-F^{-1}(\hat{V})\right]\right\} .
$$

To obtain the last term in the above equation, I used the Intermediate Value theorem to compute the integral in (6.3). Compute:

$$
\left.\frac{1}{\varepsilon} \dot{V}\right|_{V=\hat{V}}=\frac{1}{\varepsilon}\left[\left.\dot{V}\right|_{V=\hat{V}}-\left.\dot{V}\right|_{V=\bar{V}}\right] \rightarrow-\left(\frac{d \dot{V}}{d V}\right)_{V=\bar{V}}=-\left[\delta-u^{\prime}(\bar{w}) w^{\prime}(\bar{V})\right]=0 .
$$


The first equality comes from the fact that $\dot{V}=0$ at $\bar{V}$, the second equality from substituting (3.3) and $p(\bar{V})=0$, and the last equality from substituting $w^{\prime}(\bar{V})$ from (D.1). Moreover, compute:

$$
\lim _{\varepsilon \rightarrow 0}\left\{\frac{1}{\varepsilon}\left[\hat{V}-F^{-1}(\hat{V})\right]\right\}=\lim _{\varepsilon \rightarrow 0}\left\{\frac{1}{\varepsilon}\left[F^{-1}(\bar{V})-F^{-1}(\hat{V})\right]-1\right\}=\frac{1}{F^{\prime}(\bar{V})}-1 .
$$

The first equality comes from substituting $\hat{V}=\bar{V}-\varepsilon$ and $\bar{V}=F(\bar{V})$, while the second equality comes from the fact that $d F^{-1} / d V=1 / F^{\prime}$. Substituting these results and the fact $p(\bar{V})=0$ into (E.5), I obtain: $g(\bar{V})\left[\delta-\lambda_{1} p(\bar{V}) / F^{\prime}(\bar{V})\right]=0$. Thus, $g(\bar{V})=0$ if and only if $\left|\delta-\lambda_{1} p(\bar{V}) / F^{\prime}(\bar{V})\right| \neq 0$. Under this condition, continuity of $g(V)$ implies that $g^{\prime}(V)<0$ when $V$ is close to $\bar{V}$.

To check when the two matching functions in Example 2.1 satisfy the required condition for $g(\bar{V})=0$, rewrite the condition as $\left|\delta-\lambda_{1} p(\bar{V}) \frac{d F^{-1}(V)}{d V}\right|_{V=\bar{V}} \mid \neq 0$. Rewrite (3.2) as $F^{-1}(V)=V+p(V) / p^{\prime}(V)$ and differentiate it to obtain $d F^{-1}(V) / d V$. Using $p(V)=$ $M(q(V))$ and $q(V)=k / J(V)$ to compute $p^{\prime}(V)$ and $p^{\prime \prime}(V)$, I derive:

$$
p(V) \frac{d F^{-1}(V)}{d V}=2 M+\frac{M^{2}}{q M^{\prime}} \frac{J J^{\prime \prime}}{\left(J^{\prime}\right)^{2}}-\frac{M^{2}}{q M^{\prime}}\left(\frac{q M^{\prime \prime}}{M^{\prime}}+2\right) .
$$

With the CES matching function,

$$
\frac{M}{q M^{\prime}}=1-\frac{q^{\rho}}{1-\alpha}, \quad \frac{M}{q M^{\prime}}\left(\frac{q M^{\prime \prime}}{M^{\prime}}+2\right)=2-\frac{1-\rho}{1-\alpha} q^{\rho} .
$$

Because Assumption 2 requires $\rho<0$, then $\bar{q}=(1-\alpha)^{1 / \rho}$. In this case, the limit $V \rightarrow \bar{V}$ (i.e., $q \rightarrow \bar{q}$ ) implies: $M \rightarrow 0, M^{\prime} \rightarrow-\infty, \frac{M}{q M^{\prime}} \rightarrow 0$, and $-\frac{M}{q M^{\prime}}\left(\frac{q M^{\prime \prime}}{M^{\prime}}+2\right) \rightarrow 1+\rho$. Because $J, J^{\prime}$ and $J^{\prime \prime}$ are all bounded, $p(V) d F^{-1}(V) / d V \rightarrow 0$ as $V \rightarrow \bar{V}$. Then, the condition required for $g(\bar{V})=0$ is $\delta \neq 0$, which is satisfied.

With the urn-ball matching function,

$$
\begin{gathered}
\frac{M^{2}}{q M^{\prime}}=\frac{(\bar{q}-q) \ln (1-q / \bar{q})}{(\bar{q}-q) \ln (1-q / \bar{q})+q} M \\
\frac{M^{2}}{q M^{\prime}}\left(\frac{q M^{\prime \prime}}{M^{\prime}}+2\right)=-q\left\{\left[\frac{q}{(\bar{q}-q) \ln (1-q / \bar{q})+q}\right]^{2}+\frac{2}{(\bar{q}-q) \ln (1-q / \bar{q})}\right\} .
\end{gathered}
$$

The limit $V \rightarrow \bar{V}$ implies $q \rightarrow \bar{q}$ and $M \rightarrow 0$. Then, the above two expressions approach 0 and $-\bar{q}$, respectively, and so $p(V) d F^{-1}(V) / d V \rightarrow \lambda_{1} \bar{q}$. In this example, the condition required for $g(\bar{V})=0$ is $\bar{q} \neq \delta / \lambda_{1}$. QED 


\section{References}

[1] Acemoglu, D. and R. Shimer, 1999a, "Efficient unemployment insurance," Journal of Political Economy 107, 893-928.

[2] Acemoglu, D. and R. Shimer, 1999b, "Holdups and efficiency with search frictions," International Economic Review 40, 827-850.

[3] Buchinsky, M. and J. Hunt, 1999, "Wage mobility in the United States," Review of Economics and Statistics 81, 351-368.

[4] Burdett, K. and M.G. Coles, 2003, "Equilibrium wage-tenure contracts," Econometrica $71,1377-1404$.

[5] Burdett, K. and D. Mortensen, 1998, "Wage differentials, employer size, and unemployment," International Economic Review 39, 257-273.

[6] Burdett, K., Shi, S. and R. Wright, 2001, "Pricing and matching with frictions," Journal of Political Economy 109, 1060-1085.

[7] Coles, M. and J. Eeckhout, 2003, "Indeterminacy and directed search," Journal of Economic Theory 111, 2003, 265-276.

[8] Delacroix, A. and S. Shi, 2006, "Directed search on the job and the wage ladder," International Economic Review 47, 651-699.

[9] Diamond, P., 1982, "Wage determination and efficiency in search equilibrium," Review of Economic Studies 49, 217-227.

[10] Farber, H.S., 1999, "Mobility and stability," in O. Ashenfelter and D. Card (eds.) Handbook of Labor Economics, Vol. 3B, Amsterdam: Elsevier.

[11] Galenianos, M. and P. Kircher, 2005, "Directed search with multiple job applications," manuscript, University of Pennsylvania.

[12] Harris, M. and B. Holmstrom, 1982, "A theory of wage dynamics," Review of Economic Studies 49, 315-333.

[13] Hosios, A., 1990, "On the efficiency of matching and related models of search and unemployment," Review of Economic Studies 57, 279-298.

[14] Jovanovic, B., 1979, "Job matching and the theory of turnover," Journal of Political Economy 87, 972-990.

[15] Julien, B., Kennes, J. and I. King, 2000, "Bidding for labor," Review of Economic Dynamics 3, 619-649.

[16] Julien, B., Kennes, J. and I. King, 2006, "Residual wage disparity and coordination unemployment," International Economic Review 47, 961-989. 
[17] Kiefer, N.M. and G.R. Neumann, 1993, "Wage dispersion with homogeneity: the empirical equilibrium search model", in: Bunzel, et al. (eds), Panel Data and Labour Market Dynamics (pp. 57-74). Amsterdam: North Holland.

[18] Menzio, G. and S. Shi, 2007, "On-the-job search over the business cycles," preliminary work, University of Pennsylvania.

[19] Moen, E.R., 1997, "Competitive search equilibrium," Journal of Political Economy 105, 385-411.

[20] Montgomery, J.D., 1991, "Equilibrium wage dispersion and interindustry wage differentials," Quarterly Journal of Economics 106, 163-179.

[21] Mortensen, D., 1982, "Property rights and efficiency in mating, racing, and related games," American Economic Review 72, 968-979.

[22] Moscarini, G., 2005, "Job matching and the wage distribution," Econometrica 73, 481-516.

[23] Peters, M., 1984, "Bertrand equilibrium with capacity constraints and restricted mobility," Econometrica 52, 1117-1129.

[24] Peters, M., 1991, "Ex ante price offers in matching games: Non-steady state," Econometrica 59, 1425-1454.

[25] Pissarides, C., 1990, Equilibrium Unemployment Theory, Cambridge, Massachusetts: Basil Blackwell.

[26] Postel-Vinay, F. and J-M. Robin, 2002, "Equilibrium wage dispersion with worker and employer heterogeneity," Econometrica 70, 2295-2350.

[27] Shi, S., 2001, "Frictional assignment I: efficiency," Journal of Economic Theory 98, 232-260.

[28] Shi, S., 2002, "A directed search model of inequality with heterogeneous skills and skill-biased technology," Review of Economic Studies 69, 467-491.

[29] Stevens, M., 2004, "Wage-tenure contracts in a frictional labour market: firms' strategies for recruitment and retention," Review of Economic Studies 71, 535-551.

[30] van den Berg, G. and G. Ridder, 1998, "An empirical equilibrium search model of the labor market," Econometrica 66, 1183-1221. 